\title{
Three-layer supply chain in an imperfect production inventory model with two storage facilities under fuzzy rough environment
}

\author{
Amalesh Kumar Manna ${ }^{1}$, Jayanta Kumar Dey ${ }^{2 *}$ and Shyamal Kumar Mondal ${ }^{1}$
}

\author{
*Correspondence: \\ dey_jaykum@yahoo.com \\ 2 Department of Mathematics, \\ Mahishadal Raj College, Mahishadal, \\ West Bengal, 721628, India \\ Full list of author information is \\ available at the end of the article
}

\begin{abstract}
This article focuses on an imperfect production inventory model considering product reliability and reworking of imperfect items in three-layer supply chain under fuzzy rough environment. In the model, the supplier receives the raw materials, all are not of perfect quality, in a lot and delivers the items of superior quality to the manufacturer and the inferior quality items are sold at a reduced price in a single batch by the end of the cent percent screening process. The manufacturer produces a mixture of perfect and imperfect quality items. A portion of the imperfect items is transformed into perfect quality items after rework. Another portion of imperfect items, termed as 'less perfect quality items,' is sold at a reduced price to the retailer, and the portion which cannot be either transformed to the perfect quality items or sold at a reduce price is being rejected. Here, retailer purchases both the perfect and imperfect quality items from the manufacturer to sell the items to the customers through his/her respective showrooms of finite capacities. A secondary warehouse of infinite capacity is hired by the retailer on rental basis to store the excess quantity of perfect quality items. This model considers the impact of business strategies such as optimal order size of raw materials, production rate, and unit production cost in different sectors in a collaborating marketing system that can be used in the industry, like textile, footwear, and electronics goods. An analytical method has been used to optimize the production rate and raw material order size for maximization of the average profit of the integrated model. Finally, a numerical example is given to illustrate the model.
\end{abstract}

Keywords: Inventory; Three-layer supply chain; Imperfect production; Two-warehouse; Stock-dependent demand; Fuzzy rough parameters

\section{Introduction}

Business organizations all over the world are striving hard to evolve strategies to survive in the era of competition ushered in by globalization. Supply chain management (SCM) is one such strategy. It is an effective methodology and presents an integrated approach to resolve issues in sourcing customer service, demand flow, and distribution. The focus is on the customer. The results are in the form of reduced operational costs, improved flow of supplies, reduction in delays of production, and increased customer satisfaction. While the goal of supply chain management is to reduce cost of producing and reaching the finished products to the customers, inventory control is the means to achieve the

(C) 2014 Manna et al.; licensee Springer. This is an Open Access article distributed under the terms of the Creative Commons

Attribution License (http://creativecommons.org/licenses/by/4.0), which permits unrestricted use, distribution, and reproduction in any medium, provided the original work is properly credited. 
goal. Researchers as well as practitioners in manufacturing industries have given importance to develop inventory control problems in supply chain management. All steps from supply of raw materials to finished products can be included into a supply chain, connecting raw materials supplier, manufacturer, retailer, and finally, customers. Recent reviews on supply chain management are provided by Weng [1], Munson and Rosenblatt [2], Yang and Wee [3], Khouja [4], Yao et al. [5], Chaharsooghi et al. [6], Wang et al. [7], and others.

Nowadays, it is common to all industries that a certain percentage of produced or ordered items are a mixture of perfect and imperfect quality. It is also important to a supply manager of any organization to control and maintain the inventories of perfect and imperfect quality items. Salameh and Jaber [8] developed an inventory model for imperfect quality items using the economic production quantity (EPQ)/economic order quantity (EOQ) formulae and assumed that inferior quality items are sold as a single batch at the end of the total screening process. Thereafter, Goyal and Cardenas-Barron [9] extended the idea of Salameh and Jaber's [8] model and proposed a practical approach to determine EPQ for items with imperfect quality. Yu et al. [10] generalized the models of Salameh and Jaber [8], incorporating deterioration and partial back ordering. Liu and Yang [11] investigated a single-stage production system with imperfect process delivering two types of defects: reworkable and non-reworkable items. The reworkable items are sent for reworking, whereas non-reworkable items are immediately discarded from the system. They determined the optimal lot size that maximized the expected total profit over the expected time length of the production cycle. Panda and Maiti [12] represented a geometric programming approach for multi-item inventory models with price-dependent demand under flexibility and reliability with imprecise space constraint. Ma et al. [13] considered the effects of imperfect production processes and the decision on whether and when to implement a screening process for defective items generated during a production run. Sana [14] develops two inventory models in an imperfect production system and showed that the inferior quality items could be reworked at a cost where overall production inventory costs could be reduced significantly. Sana [15] extended the idea of imperfect production process in three-layer supply chain management system.

Inventory management is generally attracted for large stock for several reasons: an attractive price discount for bulk purchase, the replenishment cost including transportation cost is higher than the inventory related cost, the demand of an item is very high, and so on. Therefore, due to space limitation of showroom, one (or sometimes more than one) warehouse(s) is hired on rental basis to store the excess items. The secondary warehouse (SW) may be located away from the showroom or nearer to the showroom. The actual service to the customer is done at the showroom only. Hartely [16] first introduced the basic two warehouses problem in his book Operations Research - A Managerial Emphasis. After Hartely [16], a number of research papers have been published by different authors. Among them, the work done by Sarma [17], Dave [18], Goswami and Chaudhuri [19], Pakkala and Achary [20], Bhunia and Maiti [21], Benkherouf [22], Zhou [23], Kar et al. [24], and Chung and Huang [25] are worth mentioning. Dey et al. [26] considered a finitetime horizon inventory problem for a deteriorating item having two separate warehouses with interval-valued lead time under inflation and a time value of money. Liang and Zhou [27] investigated a two-warehouse inventory model for deteriorating items under conditionally permissible delay in payments. Hariga [28] proposed an EOQ model with 
multiple storage facilities where both owned and rented warehouses had limited stock capacity. They assumed the rented warehouse had higher unit holding costs than the own warehouse but offered better preservation resulting in a lower rate of deterioration for the goods than in the own warehouse.

Dubois and Prade [29] first studied the fuzzification problem of rough sets. Furthermore, Morsi and Yakout [30] defined the upper and lower approximations of the fuzzy sets with respect to a fuzzy min-similarity relation. Additionally, Radzikowska and Kerre [31], Xu and Zhou [32], Liu and Sai [33], Chen [34], and others generalized the above definitions of the fuzzy rough set to a more general case. Different types of uncertainty such as randomness, fuzziness, and roughness are common factors in any production inventory problem. But in some problems in production inventory system, both fuzziness and roughness occur simultaneously. In many cases, it is found that some inventory parameters involve both the fuzzy and rough uncertainties. For example, the inventory related costs holding cost, set-up cost, idle costs, etc. depend on several factors such as bank interest, inflation, etc. which are uncertain in fuzzy rough sense. To be more specific, inventory holding cost is sometimes represented by a fuzzy number and it depends on the storage amount which may be imprecise and range within an interval due to several factors such as scarcity of storage space, market fluctuation, and human estimation thought process, i.e., it may be represented by a rough set.

In this paper, a supply chain model consisting of supplier, manufacturer, and retailer has been considered. Here supplier receives the raw materials in a lot and then the superior quality items of the raw materials are sold at a higher price to the manufacturer after the screening the imperfect raw materials as well as inferior quality items of the raw materials are also sold to another manufacturer at a reduced price in a single batch by the end of cent percent screening process. A mixture of perfect and imperfect quality items is produced by the manufacturer. After some rework, some repairable portion of imperfect quality items are transformed into perfect quality items and some of non-repairable portion of imperfect items are sold with reduced price to the retailer. Retailer purchases both perfect and imperfect quality items and sells both items to the customers through his/her respective showrooms of finite capacities at a market place.

It is assumed that the customers' demand is stock dependent and selling price dependent for the perfect quality items and less perfect items, respectively. Since the storage space of the showroom for perfect quality items is limited due to space problem and the demand of the corresponding items is stock dependent, hence a secondary warehouse is hired by the retailer on rental basis to store the excess amount of perfect quality items and these items are continuously transferred to the showroom concerned. The literature suggests that the holding cost of secondary warehouse per unit item per unit time is more than the holding cost of the showroom due to the preservation cost for maintaining the quality of the product and other costs related to holding large quantity of the product in the secondary warehouse. But in this paper, it has been considered that the holding cost of perfect quality items in the secondary warehouse is less than the holding cost of the showroom as the nature of the items are non-deteriorating and so having no preservation cost. The perfect quality items are transported to the showroom via secondary warehouse, and the less perfect quality items are directly transported to the related showroom. For this purpose, transportation cost is incurred to transport both quality items at the respective showrooms from the production center. Due to complexity of environment, inventory 
holding costs, idle costs, set-up costs, and transportation costs are considered as fuzzy rough type and these are reduced to crisp ones using fuzzy rough expectation. In order to optimize the production rate and raw material order size (the decision variables), the average profit function of the manufacturer is maximized as the manufacturer acts as a leader (Stakelberg approach), and the supplier as well as retailer are the followers of that chain. The decision variables are also optimized by maximizing the integrated average profit function of the chain. Finally, a comparative study has been made between both Stakelberg and integrated approaches. A numerical example is provided to illustrate the feasibility of the model.

\section{Necessary knowledge about fuzzy-rough (Fu-Ro) set}

In this section, we discuss some basic concepts, theorems, and lemmas on fuzzy rough theory by $\mathrm{Xu}$ and $\mathrm{Zhou}$ [32]. These results are crucial for the remainder of this paper.

Definition 1. Xu and Zhou [32] proposed some definitions and discussed some important properties of fuzzy rough variable. Let $U$ be a universe, and $X$ be a set representing a concept. Then, its lower and upper approximation is defined by

$$
\underline{X}=\{x \in U \mid R(x) \subset X\} \text { and } \bar{X}=\bigcup_{x \in X} R(x) \text { respectively. }
$$

Definition 2. The collection of all sets having the same lower and upper approximations is called a rough set, denoted by $(\underline{X}, \bar{X})$. The figure of the rough set is depicted in Figure 1.

Example 1. Let $\xi$ focus on the continuous set in the one dimension real space $R$. There are still some vague sets which cannot be directly fixed and need to be described by the rough approximation. Let set $R$ be the universe, a similarity relation ! is defined as $a \simeq b$ if and only if $|a-b| \leq 10$. Let us define for the set $[20,50]$, its lower approximation $\underline{[20,50]}=[30,40]$ and its upper approximation $\overline{[20,50]}=[10,60]$. Then the upper and lower approximations of the set $[20,50]$ make up a rough set $([30,40],[10,60])$ which is the collection of all sets having the same lower approximation $[30,40]$ and upper approximation $[10,60]$.

Definition 3. A fuzzy rough variable $\xi$ is a fuzzy variable with uncertain parameter $\rho \in X$, where $X$ is approximated by $(\underline{X}, \bar{X})$ according to the similarity relation $R$, namely, $\underline{X} \subseteq X \subseteq \bar{X}$.

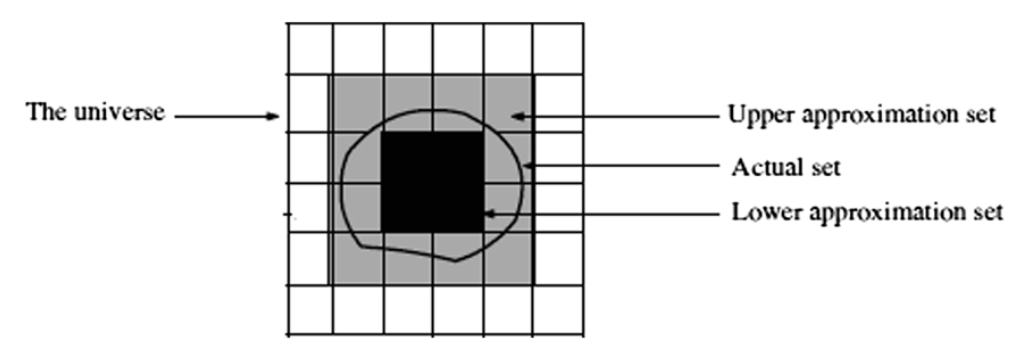

Figure 1 A rough set. 
For convenience, we usually denote $\rho \vdash(\underline{X}, \bar{X})_{R}$ expressing that $\rho$ is in some set $A$ which is approximated by $(\underline{X}, \bar{X})$ according to the similarity relation $R$, namely, $\underline{X} \subseteq A \subseteq \bar{X}$.

Example 2. Let us consider the LR fuzzy variable $\xi$ with the following membership function:

$$
\mu_{\xi}(x)= \begin{cases}L\left(\frac{\rho-x}{\alpha}\right) & \text { if } \rho-\alpha<x<\rho \\ 1 & \text { if } x=\rho \\ L\left(\frac{x-\rho}{\beta}\right) & \text { if } \rho<x<\rho+\beta\end{cases}
$$

where $L(x)=1-x$ and $\rho \vdash([1,2],[0,3])$, then $\xi$ is a fuzzy rough variable.

Theorem 1. If fuzzy rough variables $\tilde{\bar{c}}_{i j}$ are defined as $\tilde{\bar{c}}_{i j}(\lambda)=\left(\bar{c}_{i j 1}, \bar{c}_{i j 2}, \bar{c}_{i j 3}, \bar{c}_{i j 4}\right)$ with $\bar{c}_{i j t} \vdash\left(\left[c_{i j t 2}, c_{i j t 3}\right],\left[c_{i j t 1}, c_{i j t 4}\right]\right)$, for $i=1,2, \ldots, m, j=1,2, \ldots, n, t=1,2,3,4, x=$ $\left(x_{1}, x_{2}, \ldots, x_{m}\right), 0 \leq c_{i j t 1} \leq c_{i j t 2}<c_{i j t 3} \leq c_{i j t 4}$, then $E\left[\tilde{\bar{c}}_{1}^{T} x\right], E\left[\tilde{\bar{c}}_{2}^{T} x\right], \ldots, E\left[\tilde{\bar{c}}_{n}^{T} x\right]$ is respectively equivalent to

$$
\frac{1}{16} \sum_{j=1}^{n} \sum_{t=1}^{4} \sum_{k=1}^{4} c_{1 j t k} x_{j}, \frac{1}{16} \sum_{j=1}^{n} \sum_{t=1}^{4} \sum_{k=1}^{4} c_{2 j t k} x_{j}, \ldots ., \frac{1}{16} \sum_{j=1}^{n} \sum_{t=1}^{4} \sum_{k=1}^{4} c_{n j t k} x_{j} .
$$

Proof. The proof of the theorem is in reference Xu and Zhou [32] in page 308.

Theorem 2. If fuzzy rough variables $\tilde{\bar{a}}_{r j}, \tilde{\bar{b}}_{r}$ defined as follows, $\tilde{\bar{a}}_{r j}(\lambda)=$ $\left(\bar{a}_{r j 1}, \bar{a}_{r j 2}, \bar{a}_{r j 3}, \bar{a}_{r j 4}\right)$ with $\bar{a}_{r j t} \vdash\left(\left[a_{r j t 2}, a_{r j t 3}\right],\left[a_{r j t 1}, a_{r j t 4}\right]\right), \tilde{\bar{b}}_{r}(\lambda)=\left(\bar{b}_{r 1}, \bar{b}_{r 2}, \bar{b}_{r 3}, \bar{b}_{r 4}\right)$ with $\bar{b}_{r t} \vdash\left(\left[b_{r t 2}, b_{r j t 3}\right],\left[b_{r t 1}, b_{r t 4}\right]\right)$, for $r=1,2, \ldots, p, j=1,2, \ldots, n, t=1,2,3,4$, $0 \leq a_{r t 1} \leq a_{r t 2}<a_{r t 3} \leq a_{r t 4}, 0 \leq b_{r t 1} \leq b_{r t 2}<b_{r t 3} \leq b_{r t 4}$. Then $E\left[\tilde{\bar{a}}_{r j}^{T} x\right] \leq E\left[\tilde{\bar{b}}_{r j}\right], r=1,2, \ldots, p$ is equivalent to

$$
\frac{1}{16} \sum_{j=1}^{n} \sum_{t=1}^{4} \sum_{k=1}^{4} a_{r j t k} x_{j} \leq \frac{1}{16} \sum_{t=1}^{4} \sum_{k=1}^{4} b_{r t k}, r=1,2, \ldots, p
$$

Proof. The proof of the theorem is in reference Xu and Zhou [32] in page 316.

Lemma 1. Assume that $\xi$ and $\eta$ are the introduction of variables with finite expected values. Then for any real numbers $a$ and $b$, we have

$$
E[a \xi+b \eta]=a E[\xi]+b E[\eta]
$$

Proof. The proof of the Lemma is in reference $\mathrm{Xu}$ and Zhou [32] in page 313.

\section{Single-objective Fu-Ro model}

Let us consider the following single-objective decision-making model with fuzzy rough coefficients:

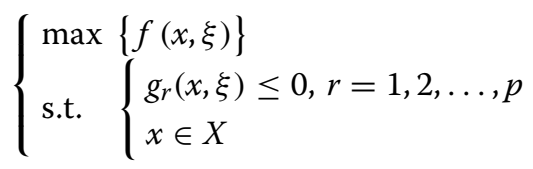


where $x$ is a $n$-dimensional decision vector, $\xi=\left(\xi_{1}, \xi_{2}, \xi_{3}, \ldots, \xi_{n}\right)$ is a Fu-Ro vector, and $f(x, \xi)$ is objective function. Because of the existence of Fu-Ro vector $\xi$, problem (3) is not well-defined, that is, the meaning of maximizing $f(x, \xi)$ is not clear and constraints $g_{r}(x, \xi) \leq 0, r=1,2, \ldots, p$ do not define a deterministic feasible set.

\section{Equivalent crisp model for single-objective problem with Fu-Ro parameters}

For the single-objective model with Fu-Ro parameters, we cannot deal with it directly, we should use some tools to make it have mathematical meaning, we then can solve it. In this subsection, we employ the expected value operator to transform the fuzzy rough model into Fu-Ro EVM, i.e., crisp model. Based on the definition of the expected value of fuzzy rough events $f(x, \xi), g_{r}(x, \xi)$ and Theorems 1 and 2, the Fu-Ro EVM is proposed as follows:

$$
\begin{cases}\max & \mathrm{E}[f(x, \xi)] \\
\text { s.t. } & \left\{\begin{array}{l}
E\left[g_{r}(x, \xi)\right] \leq 0, r=1,2, \ldots, p \\
x \in X
\end{array}\right.\end{cases}
$$

where $x$ is the $n$-dimensional decision vector and $\xi$ is the $n$-dimensional fuzzy rough variable.

\section{Assumptions and notations}

The following notations and assumptions has been used in develop the proposed models.

\section{Notations}

For convenience, the following notations are used throughout the entire paper:

$R$ Replenishment lot size of the supplier

$P$ Production rate for the manufacturer which is also the demand rate of supplier

$x$ Screening rate of supplier

$\theta$ Percentage of inferior quality items in each lot received by the supplier

$t_{1}$ Cycle length of supplier

$t^{\prime} \quad$ Total screening time of $R$ units order per cycle

$A_{\mathrm{s}}$ Set-up cost of supplier

$c_{\mathrm{S}}$ Purchase cost per unit item of supplier

$h_{\mathrm{s}}$ Holding cost per unit item for per unit time for supplier

$I_{\mathrm{CS}}$ Cost per unit idle time of supplier

$s_{\mathrm{C}}$ Screening cost per unit item

$w_{\mathrm{S}}$ Selling price per unit of superior quality item for supplier

$w_{\mathrm{S}}^{\prime} \quad$ Selling price per unit of inferior quality item for supplier

$\beta$ Percentage of imperfect quality items suitable for rework to make perfect items

$\gamma$ Percentage of imperfect items which are suitable for sale through reduction

$q_{1 m}(t)$ Inventory level of perfect quality items for the manufacturer at any time $t$

$q_{2 m}(t)$ Inventory level of less perfect quality items for the manufacturer at any time $t$

$D_{\mathrm{r}}$ Demand rate of the retailer for perfect quality items

$D_{\mathrm{r}}^{\prime}$ Demand rate of the retailer for less perfect quality items

$A_{\mathrm{m}}$ Set-up cost of manufacturer

$h_{\mathrm{m}}$ Holding cost per unit item for per unit time for perfect item in manufacturer

$h_{\mathrm{m}}^{\prime} \quad$ Holding cost per unit item for per unit time for imperfect item in manufacturer 
$I_{\mathrm{cm}}$ Cost per unit idle time of manufacturer

$I_{\mathrm{sm}}$ Inspection cost per unit item for manufacturer

$r_{\mathrm{cm}}$ Reworking cost per unit item for manufacturer

$C(P)$ Production cost per unit item

$s_{\mathrm{m}} \quad$ Selling price per unit of perfect quality items for manufacturer

$s_{\mathrm{m}}^{\prime} \quad$ Selling price per unit less perfect quality items for manufacturer

$q_{1 r}(t)$ Inventory level of perfect quality items for the retailer at any time $t$

$q_{2 r}(t)$ Inventory level of less perfect quality items for the retailer at any time $t$

$A_{\mathrm{r}}$ Set-up cost for perfect quality items of retailer

$A_{\mathrm{r}}^{\prime}$ Set-up cost for less perfect quality items of retailer

$h_{\mathrm{r}}$ Holding cost per unit item for per unit time of perfect quality items in $\mathrm{PW}_{1}$ for retailer

$h_{\mathrm{rs}}$ Holding cost per unit item for per unit time of perfect quality items for the retailer in secondary ware house

$h_{\mathrm{r}}^{\prime} \quad$ Holding cost per unit item for per unit time of less perfect quality items in $\mathrm{PW}_{2}$ for retailer

$s_{\mathrm{r}} \quad$ Selling price per unit of perfect quality items for retailer $\mathrm{PW}_{1}$

$s_{\mathrm{r}}^{\prime} \quad$ Selling price per unit of less perfect quality items for retailer $\mathrm{PW}_{2}$

$c_{\text {tr }}$ Transportation cost perfect item for retailer

$c_{\mathrm{tr}}^{\prime}$ Transportation cost of less perfect quality items for retailer

$S$ Capacity of secondary warehouse

$W_{j}$ Capacity of $\mathrm{PW}_{j}(j=1,2)$.

$D_{\mathrm{c}}$ Demand rate of customer for perfect quality items $\mathrm{PW}_{1}$

$D_{\text {c }}^{\prime}$ Demand rate of customer for less perfect quality items $\mathrm{PW}_{2}$

$\simeq$ Denotes the fuzzy rough parameters.

\section{Assumptions}

The following assumptions are used throughout the entire paper.

(i) Joint effect of supplier, manufacturer, and retailer is considered in a supply chain management.

(ii) Model is developed for single-item products and lead time is negligible.

(iii) Production rate is a decision variable.

(iv) Demand for perfect quality items is deterministic and function of current stock level.

(v) Replenishment rate of manufacture is instantaneously infinite but its size is finite.

(vi) Unit production cost is a function of production rate.

(vii) The manufacturer has ignored the machine breakdown.

(viii) Cost of idle times of supplier and manufacturer is taken into account.

(ix) Showrooms $P W_{1}$ and $P W_{2}$ of the retailer are adjacent.

\section{Formulation of three-layer supply chain production inventory model}

Block diagram and pictorial representation of the proposed supply chain production inventory model are respectively depicted in Figures 2 and 3. Formulation of the model for supplier, manufacturer, and retailer are given in the subsections 'Formulation of the supplier,' 'Formulation of the manufacturer,' and 'Formulation of the retailer,' respectively. 


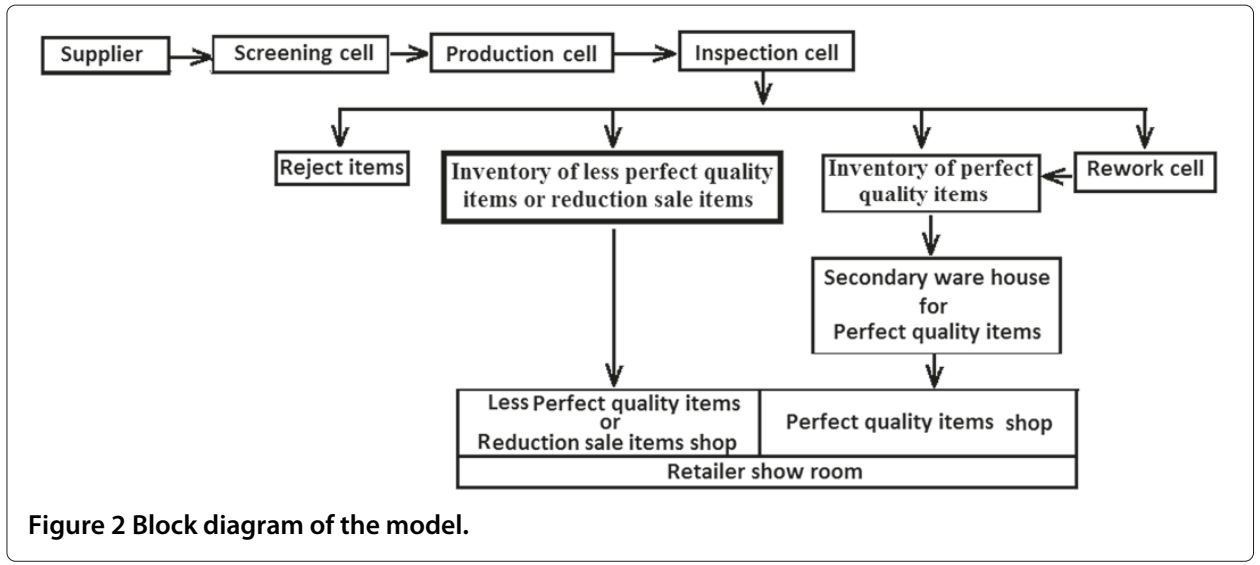

\section{Formulation of the supplier}

Here, let $R$ be the lot-size received by the supplier at $t=0$. A screening process of the lot is conducted at a rate of $x$ units per unit time and $t^{\prime}$ be the total screening time of $R$ units. Defective items are kept in stock and sold prior to receiving the next shipment as a single batch at a discounted price of $w_{\mathrm{s}}^{\prime}$ per unit. $R \theta$ is the number of inferior quality items withdrawn from inventory, and $t_{1}$ is the cycle length of the supplier. The number of superior quality items in each lot, denoted by $N(R, \theta)$, is given by

$$
N(R, \theta)=(R-R \theta)
$$

Supplier supplies the superior quality items as raw materials to the manufacturer at a rate $P$ up to the time $t_{1}$ and to avoid shortages; it is assumed that the number of superior quality items $N(r, \theta)$ is at least equal to the demand during screening time $t^{\prime}$, i.e.,

$$
N(R, \theta) \geq P t^{\prime}
$$

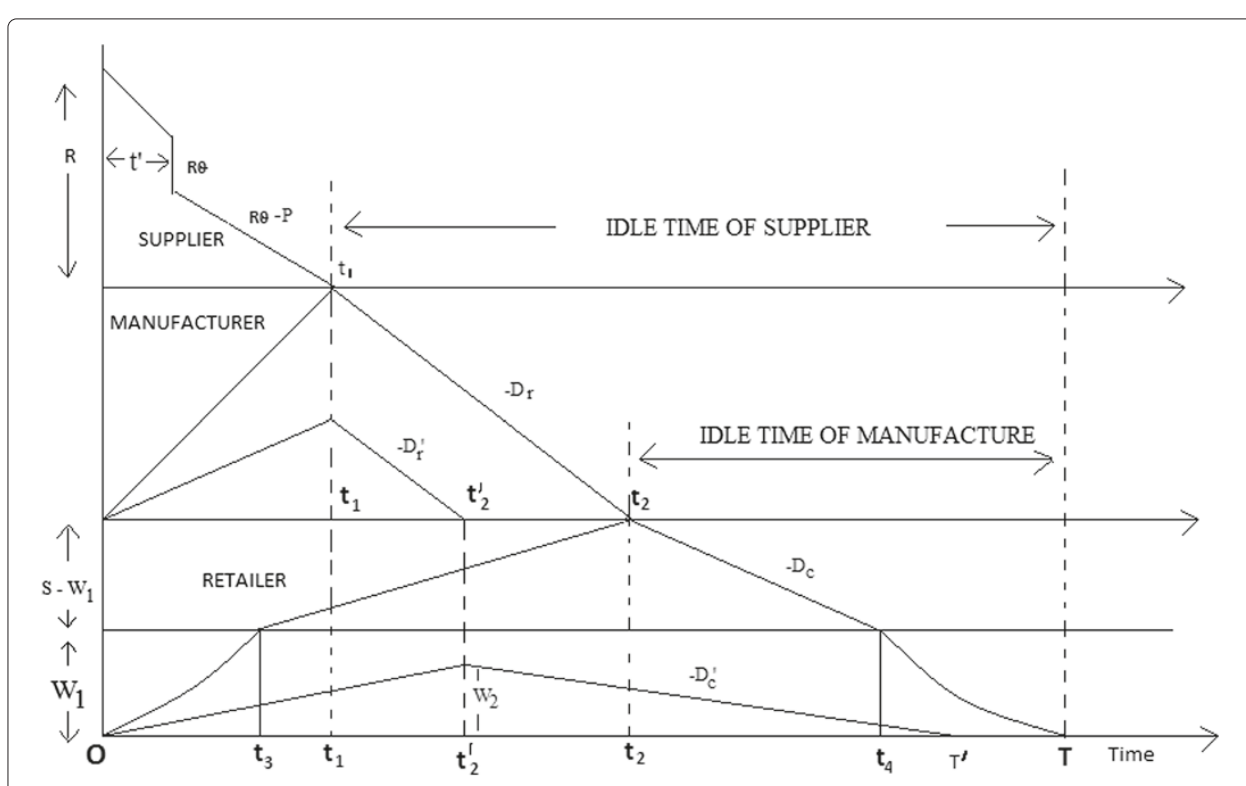

Figure 3 Pictorial representation of inventory situation of the integrated model. 
Substituting Equation 3 in Equation 4 and replacing $t^{\prime}$ by $\frac{R}{x}$, the value of $R$ is restricted to $R \leq 1-\frac{P}{x}$.

Sales revenue from superior quality items per cycle $=w_{\mathrm{s}}(R-R \theta)$.

Sales revenue from inferior quality items per cycle $=w_{\mathrm{s}}^{\prime} R \theta$.

Procurement cost for the supplier per cycle $=($ Set-up cost + Purchasing cost $)=A_{\mathrm{s}}+$ $c_{\mathrm{S}} R$.

Screening cost per cycle $=s_{\mathrm{c}} R$.

Holding cost during $\left(0, t_{1}\right)=h_{\mathrm{s}}\left[\frac{(R-R \theta) t_{1}}{2}+\frac{R^{2} \theta}{x}\right]$.

Idle time cost per cycle $=I_{\mathrm{cs}}\left(T-t_{1}\right)$.

Therefore, the average profit (APS) of the supplier during $(0, T)$ is given by

APS $=\frac{1}{T}[($ Sales revenue of superior quality items $)+($ Sales revenue of inferior quality items $)$

-(Procurement cost $)-($ screening cost $)-($ Holding cost $)-($ Idle time cost $)]$

$=\frac{1}{T}\left[w_{\mathrm{s}}(R-R \theta)+w_{\mathrm{s}}^{\prime} R \theta-\left(A_{\mathrm{s}}+c_{\mathrm{s}} R\right)-s_{\mathrm{c}} R-h_{\mathrm{s}}\left\{\frac{(R-R \theta) t_{1}}{2}+\frac{R^{2} \theta}{x}\right\}-I_{\mathrm{cs}}\left(T-t_{1}\right)\right]$

$=\frac{1}{T}\left[-Z_{0 s}+Z_{1 s} R+Z_{2 s} \frac{R}{P}-Z_{3 s} \frac{R^{2}}{2 P}-Z_{4 s} R^{2}\right]$.

where $t_{1}=\frac{(R-R \theta)}{P}$ and $Z_{i s}, i=0,1,2,3,4$ are independent of $R$ and $P$. (See Appendix 1).

\section{Formulation of the manufacturer}

It is considered that a manufacturer produces some perfect and imperfect quality items at the rate of $P$ units during the period $\left(0, t_{1}\right)$, receiving the raw material from the supplier at the same rate $P$ during the period $\left(0, t_{1}\right) \cdot P e^{-\alpha t}$ and $P\left(1-e^{-\alpha t}\right)$ are respectively the expected quantity of perfect and imperfect quality items at any time $t$, where $\alpha$ be the reliability parameter given by $\alpha=\frac{\text { Number of failures }}{\text { Total units of operating hours }}$. Among the imperfect quality items, only $\beta P\left(1-e^{-\alpha t}\right)$ units per unit time become perfect quality after reworking and the portion $\gamma(1-\beta) P\left(1-e^{-\alpha t}\right)$ are less perfect quality which are sold at a reduced price to the retailer. Here $D_{\mathrm{r}}$ and $D_{\mathrm{r}}^{\prime}$ denote the demand rates of a retailer for perfect quality and less perfect quality items which are met by manufacturer during $\left(0, t_{2}\right)$ and $\left(0, t_{2}^{\prime}\right)$, respectively. The production cost $C(P)$ per unit item is considered as $C(P)=L+\frac{G}{P}+H P$, where $G$ be the total labor cost for manufacturing the items, $L$ and $H$ are respectively the material cost and tool/die cost per unit item.

\section{For perfect quality items of manufacturer}

The rate of change of inventory level of the manufacturer for perfect quality items can be represented by the following differential equations:

$$
\frac{d q_{1 m}}{d t}= \begin{cases}P e^{-\alpha t}+\beta P\left(1-e^{-\alpha t}\right)-D_{\mathrm{r}}, & 0 \leq t \leq t_{1} \\ -D_{\mathrm{r}}, & t_{1} \leq t \leq t_{2}\end{cases}
$$

with boundary conditions $q_{1 m}(t)=0$ at $t=0$ and $t=t_{2}$.

The solution of above differential equations are given by

$$
q_{1 m}(t)= \begin{cases}\frac{P}{\alpha}(1-\beta)\left(1-e^{-\alpha t}\right)+\left(P \beta-D_{\mathrm{r}}\right) t, & 0 \leq t \leq t_{1} \\ D_{\mathrm{r}}\left(t_{2}-t\right), & t_{1} \leq t \leq t_{2}\end{cases}
$$


From continuity at $t=t_{1}$, following condition is obtained:

$$
\begin{aligned}
\frac{P}{\alpha}(1-\beta)\left(1-e^{-\alpha t_{1}}\right)+\left(P \beta-D_{\mathrm{r}}\right) t_{1} & =D_{\mathrm{r}}\left(t_{2}-t_{1}\right) \\
\text { which implies } t_{2} & =\frac{P}{D_{\mathrm{r}}}\left[\frac{1}{\alpha}(1-\beta)\left(1-e^{-\alpha t_{1}}\right)+\beta t_{1}\right] .
\end{aligned}
$$

Now holding cost $\left(\mathrm{HCM}_{1}\right)$ for perfect quality items for manufacture is given by

$$
\begin{aligned}
\mathrm{HCM}_{1} & =h_{\mathrm{m}} \int_{0}^{t_{1}} q_{1 m}(t) d t+h_{\mathrm{m}} \int_{t_{1}}^{t_{2}} q_{1 m}(t) d t \\
& =h_{\mathrm{m}} \int_{0}^{t_{1}} \frac{P}{\alpha}(1-\beta)\left(1-e^{-\alpha t}\right) d t+h_{\mathrm{m}} \int_{0}^{t_{1}}\left(P \beta-D_{\mathrm{r}}\right) t d t-D_{\mathrm{r}} h_{\mathrm{m}} \int_{t_{1}}^{t_{2}} t d t \\
& =\frac{h_{\mathrm{m}} P}{\alpha}(1-\beta) t_{1}-\frac{P h_{\mathrm{m}}}{\alpha^{2}}(1-\beta)\left(1-e^{-\alpha t_{1}}\right)+h_{\mathrm{m}} P \beta \frac{t_{1}^{2}}{2}-D_{\mathrm{r}} h_{\mathrm{m}} \frac{t_{2}^{2}}{2} .
\end{aligned}
$$

and reworking cost (RCM) for manufacture:

$$
\begin{aligned}
\mathrm{RCM} & =r_{\mathrm{cm}} \int_{0}^{t_{1}} P \beta\left(1-e^{-\alpha t}\right) d t \\
& =r_{\mathrm{cm}} P \beta\left[t_{1}-\frac{1}{\alpha}\left\{1-e^{-\alpha t_{1}}\right\}\right] .
\end{aligned}
$$

\section{For less perfect quality items of manufacturer}

The rate of change of inventory level of less perfect quality items for manufacturer can be represented by the following differential equations:

$$
\frac{d q_{2 m}}{d t}= \begin{cases}\gamma(1-\beta) P\left(1-e^{-\alpha t}\right)-D_{\mathrm{r}}^{\prime}, & 0 \leq t \leq t_{1} \\ -D_{\mathrm{r}}^{\prime}, & t_{1} \leq t \leq t_{2}^{\prime}\end{cases}
$$

with boundary conditions $q_{2 m}(t)=0$ at $t=0$ and $t=t_{2}^{\prime}$.

The solution of above differential equations are given by

$$
q_{2 m}(t)= \begin{cases}-\frac{P}{\alpha} \gamma(1-\beta)\left(1-e^{-t \alpha}\right)+\left[\gamma(1-\beta) P-D_{\mathrm{r}}^{\prime}\right] t, & 0 \leq t \leq t_{1} \\ D_{\mathrm{r}}^{\prime}\left(t_{2}^{\prime}-t\right), & t_{1} \leq t \leq t_{2}^{\prime}\end{cases}
$$

From continuity at $t=t_{1}$, following condition is obtained:

$$
t_{2}^{\prime}=\frac{1}{D_{\mathrm{r}}^{\prime}}\left[-\frac{P}{\alpha} \gamma(1-\beta)\left(1-e^{-\alpha t_{1}}\right)+\gamma(1-\beta) P t_{1}\right] .
$$

Now holding cost $\left(\mathrm{HCM}_{2}\right)$ for less perfect quality items for manufacture

$$
\begin{aligned}
\mathrm{HCM}_{2} & =h_{\mathrm{m}}^{\prime} \int_{0}^{t_{1}} q_{2 m}(t) d t+h_{\mathrm{m}}^{\prime} \int_{t_{1}}^{t_{2}^{\prime}} q_{2 m}(t) d t \\
& =-h_{\mathrm{m}}^{\prime} \int_{0}^{t_{1}} \frac{P}{\alpha} \gamma(1-\beta)\left(1-e^{-\alpha t}\right) d t+h_{\mathrm{m}}^{\prime} \int_{0}^{t_{1}} \gamma\left\{P(1-\beta)-D_{\mathrm{r}}^{\prime}\right\} t d t+D_{\mathrm{r}}^{\prime} h_{\mathrm{m}}^{\prime} \int_{t_{1}}^{t_{2}^{\prime}}\left(t_{2}^{\prime}-t\right) d t \\
& =h_{\mathrm{m}}^{\prime} \frac{P \gamma}{\alpha^{2}}(1-\beta)\left[1-e^{-t_{1} \alpha}\right]-h_{\mathrm{m} \frac{P_{\gamma}}{\alpha}}^{\prime}(1-\beta) t_{1}+h_{\mathrm{m}}^{\prime}\left[P \gamma(1-\beta)-D_{\mathrm{r}}^{\prime}\right] \frac{t_{1}^{2}}{2}+h_{\mathrm{m}}^{\prime} \frac{D_{\mathrm{r}}^{\prime}}{2}\left(t_{2}^{\prime}-t_{1}\right)^{2} .
\end{aligned}
$$

Production cost for the manufacturer $=C(P) P t_{1}$.

Inspection cost $=I_{\mathrm{sm}} P t_{1}$.

Holding cost for the manufacturer $=\left[\mathrm{HCM}_{1}+\mathrm{HCM}_{2}\right]$.

Set-up cost of the manufacturer $=A_{\mathrm{m}}$.

Idle time cost for the manufacturer $=I_{\mathrm{cm}}\left(T-t_{2}\right)$.

Revenue of perfect quality items for the manufacturer $=s_{\mathrm{m}} \int_{0}^{t_{2}} D_{\mathrm{r}} d t=s_{\mathrm{m}} D_{\mathrm{r}} t_{2}$.

Revenue of less perfect quality items for the manufacturer $=s_{\mathrm{m}}^{\prime} \int_{0}^{t_{2}^{\prime}} D_{\mathrm{r}}^{\prime} d t,=s_{\mathrm{m}}^{\prime} D_{\mathrm{r}}^{\prime} t_{2}^{\prime}$. 
Average profit of manufacturer

Average profit (APM) of the manufacturer during the period $(0, T)$ is given by

$\mathrm{APM}=\frac{1}{T}[$ (Revenue of perfect and less perfect quality items for the manufacturer $)-w_{\mathrm{s}} P t_{1}$

$-($ Production cost + Inspection cost + Holding cost + Reworking cost + Idle time cost $)$

- Set-up cost]

$=\frac{1}{T}\left[\left(s_{\mathrm{m}} D_{\mathrm{r}} t_{2}+s_{\mathrm{m}}^{\prime} D_{\mathrm{r}}^{\prime} t_{2}^{\prime}\right)-w_{\mathrm{s}} P t_{1}-C(P) P t_{1}-I_{\mathrm{sm}} P t_{1}-\mathrm{HCM}_{1}-\mathrm{HCM}_{2}-\mathrm{RCM}\right.$

$\left.-A_{\mathrm{m}}-I_{\mathrm{cm}}\left(T-t_{2}\right)\right]$

$=\frac{1}{T}\left[\left(s_{\mathrm{m}} D_{\mathrm{r}} t_{2}+s_{\mathrm{m}}^{\prime} D_{\mathrm{r}}^{\prime} t_{2}^{\prime}\right)-\left\{w_{\mathrm{s}}+C(P)+I_{\mathrm{sm}}\right\} P t_{1}-r_{\mathrm{cm}}\left\{-\frac{P \beta}{\alpha}\left(1-e^{-\alpha t_{1}}\right)+P \beta t_{1}\right\}\right.$

$-A_{\mathrm{m}}-h_{\mathrm{m}}\left\{\frac{P}{\alpha}(1-\beta) t_{1}-\frac{P}{\alpha^{2}}(1-\beta)\left(1-e^{-\alpha t_{1}}\right)+P \beta \frac{t_{1}^{2}}{2}-D_{\mathrm{r}} \frac{t_{2}^{2}}{2}\right\}-I_{\mathrm{cm}}\left(T-t_{2}\right)$

$\left.-h_{\mathrm{m}}^{\prime}\left\{\frac{P}{\alpha^{2}} \gamma(1-\beta)\left(1-e^{-\alpha t_{1}}\right)-\frac{\gamma}{\alpha}(1-\beta) P t_{1}+\left(\gamma(1-\beta) P-D_{\mathrm{r}}^{\prime}\right) \frac{t_{1}^{2}}{2}+\frac{D_{\mathrm{r}}^{\prime}}{2}\left(t_{1}-t_{2}^{\prime}\right)^{2}\right\}\right]$

$=\frac{1}{T}\left[-Z_{0 m}-Z_{1 m} \frac{R}{P}+Z_{2 m} R+Z_{3 m} \frac{R^{2}}{P^{2}}+Z_{4 m} \frac{R^{2}}{P}+Z_{5 m} R^{2}-Z_{6 m} P R^{2}\right]$.

where $Z_{i m}, i=0,1,2, \ldots, 6$, are independent of $R$ and $P$. (See Appendix 1).

\section{Formulation of the retailer}

The customers' demand for both perfect and less perfect quality items are met by the retailer through the adjacent showrooms $\mathrm{PW}_{1}$ and $\mathrm{PW}_{2}$, respectively. Retailer has a secondary warehouse SW to store the excess perfect quality items which are continuously transferred to the showroom $\mathrm{PW}_{1}$. Less perfect quality items are directly transferred to the showroom $\mathrm{PW}_{2}$. Transportation cost is taken into account to transfer each items from production center to the showrooms.

\section{For perfect quality items of retailer}

In this case the demand rate $\left(D_{\mathrm{c}}\right)$ of customers at $\mathrm{PW}_{1}$ has consider as stock dependent as the following form:

$$
D_{\mathrm{c}}= \begin{cases}\alpha_{1}+\beta_{1} q_{1 r}, & 0 \leq t \leq t_{3} \\ \alpha_{1}+\beta_{1} W_{1}, & t_{3} \leq t \leq t_{4} \\ \alpha_{1}+\beta_{1} q_{1 r}, & t_{4} \leq t \leq T\end{cases}
$$

Now, the corresponding rate of change of on hand inventory of perfect quality items are given by

$$
\frac{d q_{1 r}}{d t}= \begin{cases}D_{\mathrm{r}}-D_{\mathrm{c}}, & 0 \leq t \leq t_{2} \\ -D_{\mathrm{c}}, & t_{2} \leq t \leq T\end{cases}
$$

with boundary conditions

$$
q_{1 r}(t)= \begin{cases}0, & \text { at } t=0 \\ W_{1}, & \text { at } t=t_{3} \\ S, & \text { at } t=t_{2} \\ W_{1}, & \text { at } t=t_{4} \\ 0, & \text { at } t=T\end{cases}
$$


Therefore, the solutions of above differential equations are given by

$$
q_{1 r}(t)= \begin{cases}\frac{D_{\mathrm{r}}-\alpha_{1}}{\beta_{1}}\left(1-e^{-\beta_{1} t}\right), & 0 \leq t \leq t_{3} \\ W_{1}+\left[D_{\mathrm{r}}-\left(\alpha_{1}+\beta_{1} W_{1}\right)\right]\left(t-t_{3}\right), & t_{3} \leq t \leq t_{2} \\ S-\left[\left(\alpha_{1}+\beta_{1} W_{1}\right)\right]\left(t-t_{2}\right), & t_{2} \leq t \leq t_{4} \\ -\frac{\alpha_{1}}{\beta_{1}}\left[1-e^{-\beta_{1}(t-T)}\right], & t_{4} \leq t \leq T\end{cases}
$$

Now, $q_{1 r}\left(t_{3}\right)=W_{1}$ implies $t_{3}=-\frac{1}{\beta_{1}} \log \left(1-\frac{W_{1} \beta_{1}}{D_{\mathrm{r}}-\alpha_{1}}\right)$

$$
q_{1 r}\left(t_{2}\right)=S \text { gives } t_{4}=t_{2}+\frac{S-W_{1}}{\alpha_{1}+\beta_{1} W_{1}} \text { and } W_{1}+\left[D_{\mathrm{r}}-\left(\alpha_{1}+\beta_{1} W_{1}\right)\right]\left(t_{2}-t_{3}\right)=S
$$$$
q_{1 r}\left(t_{4}\right)=W_{1} \text { implies } T=t_{4}+\frac{1}{\beta_{1}} \log \left[\left(1-\frac{D_{\mathrm{r}}}{\alpha_{1}}\right)\left(1-e^{-\beta_{1} t_{3}}\right)\right]
$$

Holding cost (HCRW) of the secondary warehouse SW is given by

$$
\begin{aligned}
\mathrm{HCRW} & =h_{\mathrm{rs}} \int_{t_{3}}^{t_{2}}\left\{q_{1 r}(t)-W_{1}\right\} d t+h_{\mathrm{rs}} \int_{t_{2}}^{t_{4}}\left\{q_{1 r}(t)-W_{1}\right\} d t \\
& =h_{\mathrm{rs}} \int_{t_{3}}^{t_{2}}\left[D_{\mathrm{r}}-\left(\alpha_{1}+\beta_{1} W_{1}\right)\right]\left(t-t_{3}\right) d t-h_{\mathrm{rs}} \int_{t_{2}}^{t_{4}}\left[\alpha_{1}+\beta_{1} W_{1}\right]\left(t-t_{4}\right) d t \\
& =\frac{h_{\mathrm{rs}}}{2}\left[\left(D_{\mathrm{r}}-\left(\alpha_{1}+\beta_{1} W_{1}\right)\right)\left(t_{2}-t_{3}\right)^{2}-\left(\alpha_{1}+\beta_{1} W_{1}\right)\left(t_{2}-t_{4}\right)^{2}\right] .
\end{aligned}
$$

Holding cost $\left(\mathrm{HCRS}_{1}\right)$ of the showroom $\mathrm{PW}_{1}$ is given by

$$
\begin{aligned}
\mathrm{HCRS}_{1} & =h_{\mathrm{r}} \int_{0}^{t_{3}} q_{1 r}(t) d t+h_{\mathrm{r}} \int_{t_{3}}^{t_{4}} W_{1} d t+\int_{t_{4}}^{T} q_{1 r}(t) d t \\
& =h_{\mathrm{r}} \int_{0}^{t_{3}} \frac{D_{\mathrm{r}}-\alpha_{1}}{\beta_{1}}\left(1-e^{-\beta_{1} t}\right) d t+h_{\mathrm{r}} \int_{t_{3}}^{t_{4}} W_{1} d t+\int_{t_{4}}^{T}-\frac{\alpha_{1}}{\beta_{1}}\left[1-e^{-\beta_{1}(t-T)}\right] d t \\
& \left.=h_{\mathrm{r}} \frac{D_{\mathrm{r}}-\alpha_{1}}{\beta_{1}}\left[t_{3}+\frac{e^{-\beta_{1} t_{3}}}{\beta_{1}}-\frac{1}{\beta_{1}}\right]+W_{1} h\right]_{\mathrm{r}}\left(t_{4}-t_{3}\right)-h_{\mathrm{r}} \frac{\alpha_{1}}{\beta_{1}}\left[\left(T-t_{4}\right)+\frac{1}{\beta_{1}}-\frac{e^{-\beta_{1}\left(t_{4}-T\right)}}{\beta_{1}}\right] .
\end{aligned}
$$

Transportation cost (TCPR) for perfect quality items of retailer is given by

$$
\begin{aligned}
\mathrm{TCPR} & =c_{\operatorname{tr}} \int_{0}^{T} D_{C} d t \\
& =c_{\operatorname{tr}}\left[\int_{0}^{t_{3}}\left(\alpha_{1}+\beta_{1} q_{1 r}\right) d t+\int_{t_{3}}^{t_{4}}\left(\alpha_{1}+\beta_{1} W_{1}\right) d t+\int_{t_{4}}^{T}\left(\alpha_{1}+\beta_{1} q_{1 r}\right) d t\right] \\
& =c_{\operatorname{tr}}\left[\left(\alpha_{1}+\beta_{1} W_{1}\right)\left(t_{4}-t_{3}\right)+D_{\mathrm{r}} t_{3}-\frac{D_{\mathrm{r}}}{\beta_{1}}+\frac{D_{\mathrm{r}}-\alpha_{1}}{\beta_{1}} e^{-\beta_{1} t_{3}}+\frac{\alpha_{1}}{\beta_{1}} e^{-\beta_{1}\left(t_{4}-T\right)}\right] .
\end{aligned}
$$


Revenue from selling perfect quality items is given by

$$
\begin{aligned}
\mathrm{REPR} & =s_{\mathrm{r}} \int_{0}^{T} D_{\mathrm{c}} d t \\
& =s_{\mathrm{r}}\left[\left(\alpha_{1}+\beta_{1} W_{1}\right)\left(t_{4}-t_{3}\right)+D_{\mathrm{r}} t_{3}-\frac{D_{\mathrm{r}}}{\beta_{1}}+\frac{D_{\mathrm{r}}-\alpha_{1}}{\beta_{1}} e^{-\beta_{1} t_{3}}+\frac{\alpha_{1}}{\beta_{1}} e^{-\beta_{1}\left(t_{4}-T\right)}\right] .
\end{aligned}
$$

Hence, the total profit of the retailer from the perfect quality items is given by

$\mathrm{TPR}_{1}=$ Revenue from selling perfect quality items - Transportation cost for perfect quality items

- Holding cost of SW - Holding cost of $\mathrm{PW}_{1}-$ Set-up cost $-s_{\mathrm{m}}(P A)_{m}$

$$
\begin{aligned}
= & s_{\mathrm{r}}\left[\left(\alpha_{1}+\beta_{1} W_{1}\right)\left(t_{4}-t_{3}\right)+D_{\mathrm{r}} t_{3}-\frac{D_{\mathrm{r}}}{\beta_{1}}+\frac{D_{\mathrm{r}}-\alpha_{1}}{\beta_{1}} e^{-\beta_{1} t_{3}}+\frac{\alpha_{1}}{\beta_{1}} e^{-\beta_{1}\left(t_{4}-T\right)}\right] \\
& -c_{\mathrm{tr}}\left[\left(\alpha_{1}+\beta_{1} W_{1}\right)\left(t_{4}-t_{3}\right)+D_{\mathrm{r}} t_{3}-\frac{D_{\mathrm{r}}}{\beta_{1}}+\frac{D_{\mathrm{r}}-\alpha_{1}}{\beta_{1}} e^{-\beta_{1} t_{3}}+\frac{\alpha_{1}}{\beta_{1}} e^{-\beta_{1}\left(t_{4}-T\right)}\right] \\
& -\frac{h_{\mathrm{rs}}}{2}\left[\left(D_{\mathrm{r}}-\left(\alpha_{1}+\beta_{1} W_{1}\right)\right)\left(t_{2}-t_{3}\right)^{2}-\left(\alpha_{1}+\beta_{1} W_{1}\right)\left(t_{2}-t_{4}\right)^{2}\right] \\
& -h_{\mathrm{r}}\left[\frac{D_{\mathrm{r}}-\alpha_{1}}{\beta_{1}}\left(t_{3}+\frac{e^{-\beta_{1} t_{3}}}{\beta_{1}}-\frac{1}{\beta_{1}}\right)+W_{1}\left(t_{4}-t_{3}\right)-\frac{\alpha_{1}}{\beta_{1}}\left\{\left(T-t_{4}\right)+\frac{1}{\beta_{1}}-\frac{e^{-\beta_{1}\left(t_{4}-T\right)}}{\beta_{1}}\right\}\right] \\
& -A_{\mathrm{r}}-s_{\mathrm{m}}\left[\frac{P}{\alpha}(1-\beta) t_{1}-\frac{P}{\alpha^{2}}(1-\beta)\left(1-e^{-\alpha t_{1}}\right)+P \beta \frac{t_{1}^{2}}{2}-D_{\mathrm{r}} \frac{t_{2}^{2}}{2}\right] .
\end{aligned}
$$

\section{For less perfect quality items of retailer}

In this case demand rate $\left(D_{\mathrm{c}}^{\prime}\right)$ of customers at $\mathrm{PW}_{2}$ is assumed as selling price dependent defined as $D_{\mathrm{c}}^{\prime}=\left(a-b s_{\mathrm{r}}^{\prime}\right)$ and corresponding change of on hand inventory of less perfect quality items are given by

$$
\frac{d q_{2 r}}{d t}= \begin{cases}D_{\mathrm{r}}^{\prime}-D_{\mathrm{c}}^{\prime}, & 0 \leq t \leq t_{2}^{\prime} \\ -D_{\mathrm{c}}^{\prime}, & t_{2}^{\prime} \leq t \leq T^{\prime}\end{cases}
$$

with boundary conditions $q_{2 r}(t)=0$ at $t=0$ and $t=T^{\prime}$.

The solution of above differential equations is given by

$$
q_{2 r}(t)= \begin{cases}\left(D_{\mathrm{r}}^{\prime}-D_{\mathrm{c}}^{\prime}\right) t, & 0 \leq t \leq t_{2}^{\prime} \\ -D_{\mathrm{c}}^{\prime}\left(t-T^{\prime}\right), & t_{2}^{\prime} \leq t \leq T^{\prime}\end{cases}
$$

From continuity condition at $t=t_{2}^{\prime}, T^{\prime}=\frac{1}{D_{\mathrm{c}}^{\prime}}\left[\gamma(1-\beta) P t_{1}-\frac{P}{\alpha^{2}} \gamma(1-\beta)\left(1-e^{-\alpha t_{1}}\right)\right]$. Also $q_{2 r}\left(t_{2}^{\prime}\right)=W_{2}$, i.e., $W_{2}=\left(1-\frac{D_{c}^{\prime}}{D_{\mathrm{r}}^{\prime}}\right)\left[-\frac{P}{\alpha} \gamma(1-\beta)\left(1-e^{-\alpha t_{1}}\right)+\gamma(1-\beta) P t_{1}\right]$. Holding cost $\left(\mathrm{HCRS}_{2}\right)$ of the showroom $\mathrm{PW}_{2}$ is given by

$$
\begin{aligned}
\mathrm{HCRS}_{2} & =h_{\mathrm{r}}^{\prime} \int_{0}^{t_{2}^{\prime}} q_{2 r}(t) d t+h_{\mathrm{r}}^{\prime} \int_{t_{2}^{\prime}}^{T^{\prime}} q_{2 r}(t) d t \\
& =h_{\mathrm{r}}^{\prime} \int_{0}^{t_{2}^{\prime}}\left\{D_{\mathrm{r}}^{\prime}-D_{\mathrm{c}}^{\prime}\right\} t d t+h_{\mathrm{r}}^{\prime} \int_{t_{2}^{\prime}}^{T^{\prime}}\left\{-D_{\mathrm{c}}^{\prime}\left(t-T^{\prime}\right)\right\} d t \\
& =\frac{h_{\mathrm{r}}^{\prime}}{2}\left[\left(D_{\mathrm{r}}^{\prime}-D_{\mathrm{c}}^{\prime}\right) t_{2}^{\prime 2}+D_{\mathrm{c}}^{\prime}\left(t_{2}^{\prime}-T^{\prime}\right)^{2}\right] .
\end{aligned}
$$


Transportation cost (TCIR) for less perfect quality items of retailer is given by

$$
\mathrm{TCIR}=c_{\mathrm{tr}}^{\prime} \int_{0}^{T^{\prime}} D_{\mathrm{c}}^{\prime} d t=c_{\mathrm{tr}}^{\prime} D_{\mathrm{c}}^{\prime} T^{\prime}
$$

Revenue (REIR) from selling less perfect quality items of retailer is given by

$$
\operatorname{REIR}=s_{\mathrm{r}}^{\prime} \int_{0}^{T^{\prime}} D_{\mathrm{c}}^{\prime} d t=s_{\mathrm{r}}^{\prime} D_{\mathrm{c}}^{\prime} T^{\prime}
$$

Total profit $\left(\mathrm{TPR}_{2}\right)$ of retailer for less perfect quality items is given by

$\mathrm{TPR}_{2}=$ Revenue from selling less perfect quality items $-($ Transportation cost for less perfect quality items $)-\left(\right.$ Holding cost of $\left.\mathrm{PW}_{2}\right)-$ Set-up cost- $s_{\mathrm{m}}^{\prime}(D A)_{\mathrm{m}}$

$$
\begin{aligned}
= & \left\{s_{\mathrm{r}}^{\prime}-c_{\mathrm{tr}}^{\prime}\right\} D_{\mathrm{c}}^{\prime} T^{\prime}-\frac{h_{\mathrm{r}}^{\prime}}{2}\left[\left(D_{\mathrm{r}}^{\prime}-D_{\mathrm{c}}^{\prime}\right) t_{2}^{\prime 2}+D_{\mathrm{c}}^{\prime}\left(t_{2}^{\prime}-T^{\prime}\right)^{2}\right]-s_{\mathrm{m}}^{\prime}\left[\frac{P \gamma}{\alpha^{2}}(1-\beta)\left(1-e^{-t_{1} \alpha}\right)\right. \\
& \left.-\frac{P \gamma}{\alpha}(1-\beta) t_{1}+\left(P \gamma(1-\beta)-D_{\mathrm{r}}^{\prime}\right) \frac{t_{1}^{2}}{2}+\frac{D_{\mathrm{r}}^{\prime}}{2}\left(t_{1}-t_{2}^{\prime}\right)^{2}\right]-A_{\mathrm{r}}^{\prime} .
\end{aligned}
$$

\section{Average profit of retailer}

Average profit (APR) of the retailer during $(0, T)$ is

$$
\begin{aligned}
\mathrm{APR}= & \frac{1}{T}\left[\mathrm{TPR}_{1}+\mathrm{TPR}_{2}\right] \\
= & \frac{1}{T}\left[s_{\mathrm{r}}\left\{\left(\alpha_{1}+\beta_{1} W_{1}\right)\left(t_{4}-t_{3}\right)+D_{\mathrm{r}} t_{3}-\frac{D_{\mathrm{r}}}{\beta_{1}}+\frac{D_{\mathrm{r}}-\alpha_{1}}{\beta_{1}} e^{-\beta_{1} t_{3}}+\frac{\alpha_{1}}{\beta_{1}} e^{-\beta_{1}\left(t_{4}-T\right)}\right\}\right. \\
& -c_{\mathrm{tr}}\left\{\left(\alpha_{1}+\beta_{1} W_{1}\right)\left(t_{4}-t_{3}\right)+D_{\mathrm{r}} t_{3}-\frac{D_{\mathrm{r}}}{\beta_{1}}+\frac{D_{\mathrm{r}}-\alpha_{1}}{\beta_{1}} e^{-\beta_{1} t_{3}}+\frac{\alpha_{1}}{\beta_{1}} e^{-\beta_{1}\left(t_{4}-T\right)}\right\} \\
& -\frac{h_{\mathrm{rs}}}{2}\left\{\left(D_{\mathrm{r}}-\left(\alpha_{1}+\beta_{1} W_{1}\right)\right)\left(t_{2}-t_{3}\right)^{2}-\left(\alpha_{1}+\beta_{1} W_{1}\right)\left(t_{2}-t_{4}\right)^{2}\right\}+s_{\mathrm{r}}^{\prime} D_{\mathrm{c}}^{\prime} T^{\prime} \\
& -c_{\mathrm{tr}}^{\prime} D_{\mathrm{c}}^{\prime} T^{\prime}-\frac{h_{\mathrm{r}}^{\prime}}{2}\left\{\left(D_{\mathrm{r}}^{\prime}-D_{\mathrm{c}}^{\prime}\right) t_{2}^{\prime 2}+D_{\mathrm{c}}^{\prime}\left(t_{2}^{\prime}-T^{\prime}\right)^{2}\right\}-h_{\mathrm{r}}\left\{\frac { D _ { \mathrm { r } } - \alpha _ { 1 } } { \beta _ { 1 } } \left(t_{3}+\frac{e^{-\beta_{1} t_{3}}}{\beta_{1}}\right.\right. \\
& \left.\left.-\frac{1}{\beta_{1}}\right)+W_{1}\left(t_{4}-t_{3}\right)-\frac{\alpha_{1}}{\beta_{1}}\left\{\left(T-t_{4}\right)+\frac{1}{\beta_{1}}-\frac{e^{-\beta_{1}\left(t_{4}-T\right)}}{\beta_{1}}\right\}\right\}-s_{\mathrm{m}}\left\{\frac{P}{\alpha}(1-\beta) t_{1}\right. \\
& \left.-\frac{P}{\alpha^{2}}(1-\beta)\left(1-e^{-\alpha t_{1}}\right)+P \beta \frac{t_{1}^{2}}{2}-D_{\mathrm{r}} \frac{t_{2}^{2}}{2}\right\}-s_{\mathrm{m}}^{\prime}\left\{\frac{P \gamma}{\alpha^{2}}(1-\beta)\left(1-e^{-t_{1} \alpha}\right)\right. \\
& \left.-\frac{P \gamma}{\alpha}(1-\beta) t_{1}+\left(P \gamma(1-\beta)-D_{\mathrm{r}}^{\prime}\right) \frac{t_{1}^{2}}{2}+\frac{D_{\mathrm{r}}^{\prime}}{2}\left(t_{1}-t_{2}^{\prime}\right)^{2}\right\}-A_{\mathrm{r}}-A_{\mathrm{r}}^{\prime} \\
= & \frac{1}{T}\left[Z_{0 r}+Z_{1 r} R+Z_{2 r} \frac{R^{2}}{2 P}+Z_{3 r} R^{2}+Z_{4 r} \frac{R^{3}}{P}+Z_{5 r} \frac{R^{4}}{P^{2}}\right] \cdot
\end{aligned}
$$

where $Z_{i r}, i=0,1, \ldots, 5$ are independent of $R$ and $P$. (See Appendix 1). 


\section{Integrated average profit}

Average profit (IAP) for the Integrated Model during $(0, T)$ is

$$
\begin{aligned}
\mathrm{IAP} & =[\mathrm{APS}+\mathrm{APM}+\mathrm{APR}] \\
& =\frac{1}{T}\left[Z_{0}+Z_{1} \frac{R}{P}+Z_{2} R-Z_{3} \frac{R^{2}}{2 P}+Z_{4} \frac{R^{2}}{P^{2}}-Z_{5} P R^{2}-Z_{6} R^{2}+Z_{7} \frac{R^{3}}{P}+Z_{8} \frac{R^{4}}{P^{2}}\right]
\end{aligned}
$$

where $Z_{i}, i=0,1, \ldots, 8$ are independent of $R$ and $P$. (See Appendix 1).

\section{In fuzzy rough environment}

In this environment, all holding cost, idle cost, set-up cost, and transportation cost have been considered fuzzy-rough parameters. Then the corresponding fuzzy-rough objective functions for supplier, manufacturer, and retailer are given by

$$
\begin{aligned}
\mathrm{A} \tilde{\overline{\mathrm{PS}}} & =\frac{1}{T}\left[-\tilde{\bar{Z}}_{0 s}+\tilde{\bar{Z}}_{1 s} R+\tilde{\bar{Z}}_{2 s} \frac{R}{P}-\tilde{\bar{Z}}_{3 s} \frac{R^{2}}{2 P}-\tilde{\bar{Z}}_{4 s} R^{2}\right] \\
\mathrm{A} \tilde{\bar{P}} \mathrm{M} & =\frac{1}{T}\left[-\tilde{\bar{Z}}_{0 m}-\tilde{\bar{Z}}_{1 m} \frac{R}{P}+\tilde{\bar{Z}}_{2 m} R+\tilde{\bar{Z}}_{3 m} \frac{R^{2}}{P^{2}}+\tilde{\bar{Z}}_{4 m} \frac{R^{2}}{P}+\tilde{\bar{Z}}_{5 m} R^{2}-\tilde{\bar{Z}}_{6 m} P R^{2}\right] \\
\text { and A } \tilde{\bar{P}} \mathrm{R} & =\frac{1}{T}\left[\tilde{\bar{Z}}_{0 r}+\tilde{\bar{Z}}_{1 r} R+\tilde{\bar{Z}}_{2 r} \frac{R^{2}}{2 P}+\tilde{\bar{Z}}_{3 r} R^{2}+\tilde{\bar{Z}}_{4 r} \frac{R^{3}}{P}+\tilde{\bar{Z}}_{5 r} \frac{R^{4}}{P^{2}}\right]
\end{aligned}
$$

Also, the fuzzy-rough objective functions for integrated model is given by

$$
\begin{aligned}
\mathrm{I} \tilde{\overline{\mathrm{A}}} \mathrm{P} & =[\mathrm{A} \tilde{\overline{\mathrm{P}}}+\mathrm{A} \tilde{\bar{P}} \mathrm{M}+\mathrm{A} \tilde{\bar{P}} \mathrm{R}] \\
& =\frac{1}{T}\left[\tilde{\bar{Z}}_{0}+\tilde{\bar{Z}}_{1} \frac{R}{P}+\tilde{\bar{Z}}_{2} R-\tilde{\bar{Z}}_{3} \frac{R^{2}}{2 P}+\tilde{\bar{Z}}_{4} \frac{R^{2}}{P^{2}}-\tilde{\bar{Z}}_{5} P R^{2}-\tilde{\bar{Z}}_{6} R^{2}+\tilde{\bar{Z}}_{7} \frac{R^{3}}{P}+\tilde{\bar{Z}}_{8} \frac{R^{4}}{P^{2}}\right]
\end{aligned}
$$

where fuzzy rough parameters $\tilde{\overline{\bar{h}}}_{\mathrm{s}}, \tilde{\overline{\bar{h}}}_{\mathrm{m}},{\tilde{\overline{h_{\mathrm{m}}}}}^{\prime}, \tilde{\overline{\bar{h}}}_{\mathrm{r}},{\tilde{\overline{h_{\mathrm{r}}}}}_{\mathrm{r}}, \tilde{\bar{h}}_{\mathrm{rs}}, \tilde{\bar{A}}_{\mathrm{s}}, \tilde{\bar{A}}_{\mathrm{m}}, \tilde{\overline{\bar{A}}}_{\mathrm{r}}, \tilde{\bar{I}}_{\mathrm{cs}}, \tilde{\bar{I}}_{\mathrm{cm}}, \tilde{\overline{\bar{c}}}_{\mathrm{tp}},{\tilde{\overline{c^{\prime}}}}_{\mathrm{tp}}^{\prime}$ are defined as follows:

$$
\tilde{\bar{h}}_{\mathrm{s}}=\left(\bar{h}_{\mathrm{s} 1}, \bar{h}_{\mathrm{s} 2}, \bar{h}_{\mathrm{s} 3}, \bar{h}_{\mathrm{s} 4}\right) \text { with } \bar{h}_{\mathrm{st}} \vdash\left(\left[h_{\mathrm{st} 2}, h_{\mathrm{st} 3}\right],\left[h_{\mathrm{st} 1}, h_{\mathrm{st} 4}\right]\right), 0 \leq h_{\mathrm{st} 1} \leq h_{\mathrm{st} 2}<
$$
$h_{\mathrm{st} 3} \leq h_{\mathrm{st} 4}, \tilde{\bar{h}}_{\mathrm{m}}=\left(\bar{h}_{\mathrm{m} 1}, \bar{h}_{\mathrm{m} 2}, \bar{h}_{\mathrm{m} 3}, \bar{h}_{\mathrm{m} 4}\right)$ with $\bar{h}_{\mathrm{mt}} \vdash\left(\left[h_{\mathrm{mt} 2}, h_{\mathrm{mt} 3}\right],\left[h_{\mathrm{mt} 1}, h_{\mathrm{mt} 4}\right]\right), 0 \leq$ $h_{\mathrm{mt} 1} \leq h_{\mathrm{mt} 2}<h_{\mathrm{mt} 3} \leq h_{\mathrm{mt} 4} \cdot{\tilde{\overline{h_{\mathrm{m}}^{\prime}}}}^{\prime}=\left({\overline{h_{\mathrm{m} 1}^{\prime}}},{\overline{h^{\prime}}}_{\mathrm{m} 2},{\overline{h^{\prime}}}_{\mathrm{m} 3},{\overline{h^{\prime}}}_{\mathrm{m} 4}\right)$ with ${\overline{h^{\prime}}}_{\mathrm{mt}} \vdash\left(\left[h_{\mathrm{mt} 2}^{\prime}, h_{\mathrm{mt} 3}^{\prime}\right]\right.$, $\left.\left[h_{\mathrm{mt} 1}^{\prime}, h_{\mathrm{mt} 4}^{\prime}\right]\right), 0 \leq h_{\mathrm{mt} 1}^{\prime} \leq h_{\mathrm{mt} 2}^{\prime}<h_{\mathrm{mt} 3}^{\prime} \leq h_{\mathrm{mt} 4}^{\prime} \cdot \tilde{\bar{h}}_{\mathrm{r}}=\left(\bar{h}_{\mathrm{r} 1}, \bar{h}_{\mathrm{r} 2}, \bar{h}_{\mathrm{r} 3}, \bar{h}_{\mathrm{r} 4}\right)$ with $\bar{h}_{\mathrm{rt}} \vdash$ $\left(\left[h_{\mathrm{rt} 2}, h_{\mathrm{rt} 3}\right],\left[h_{\mathrm{rt} 1}, h_{\mathrm{rt} 4}\right]\right), 0 \leq h_{\mathrm{rt} 1} \leq h_{\mathrm{rt} 2}<h_{\mathrm{rt} 3} \leq h_{\mathrm{rt} 4} \cdot{\tilde{\overline{h_{\mathrm{r}}^{\prime}}}}^{\prime}=\left({\overline{h^{\prime}}}_{\mathrm{r} 1},{\overline{h^{\prime}}}_{\mathrm{r} 2},{\overline{h^{\prime}}}_{\mathrm{r} 3},{\overline{h^{\prime}}}_{\mathrm{r} 4}\right)$ with ${\overline{h^{\prime}}}_{\mathrm{rt}} \vdash\left(\left[h_{\mathrm{rt} 2}^{\prime}, h_{\mathrm{rt} 3}^{\prime}\right],\left[h_{\mathrm{rt} 1}^{\prime}, h_{\mathrm{rt} 4}^{\prime}\right]\right), 0 \leq h_{\mathrm{rt} 1}^{\prime} \leq h_{\mathrm{rt} 2}^{\prime}<h_{\mathrm{rt} 3}^{\prime} \leq h_{\mathrm{rt} 4}^{\prime} \cdot \tilde{\bar{h}}_{\mathrm{rs}}=\left(\bar{h}_{\mathrm{rs} 1}, \bar{h}_{\mathrm{rs} 2}, \bar{h}_{\mathrm{rs} 3}, \bar{h}_{r s 4}\right)$ with $\bar{h}_{\text {rst }} \vdash\left(\left[h_{\mathrm{rst} 2}, h_{\mathrm{rst} 3}\right],\left[h_{\mathrm{rst} 1}, h_{\mathrm{rst} 4}\right]\right), 0 \leq h_{\mathrm{rst} 1} \leq h_{\mathrm{rst} 2}<h_{\mathrm{rst} 3} \leq h_{\mathrm{rst} 4} \cdot \tilde{\overline{\bar{A}}}_{\mathrm{s}}=$ $\left(\bar{A}_{\mathrm{s} 1}, \bar{A}_{\mathrm{s} 2}, \bar{A}_{\mathrm{s} 3}, \bar{A}_{\mathrm{s} 4}\right)$ with $\bar{A}_{\mathrm{st}} \vdash\left(\left[A_{\mathrm{st} 2}, A_{\mathrm{st} 3}\right],\left[A_{\mathrm{st} 1}, A_{\mathrm{st} 4}\right]\right), 0 \leq A_{\mathrm{st} 1} \leq A_{\mathrm{st} 2}<A_{\mathrm{st} 3} \leq A_{\mathrm{st} 4}$. $\tilde{\bar{A}}_{\mathrm{m}}=\left(\bar{A}_{\mathrm{m} 1}, \bar{A}_{\mathrm{m} 2}, \bar{A}_{\mathrm{m} 3}, \bar{A}_{\mathrm{m} 4}\right)$ with $\bar{A}_{\mathrm{mt}} \vdash\left(\left[A_{\mathrm{mt} 2}, A_{\mathrm{mt} 3}\right],\left[A_{\mathrm{mt} 1}, A_{\mathrm{mt} 4}\right]\right), 0 \leq A_{\mathrm{mt} 1} \leq A_{\mathrm{mt} 2}<$ $A_{\mathrm{mt} 3} \leq A_{\mathrm{mt} 4} \cdot \tilde{\bar{A}}_{\mathrm{r}}=\left(\bar{A}_{\mathrm{r} 1}, \bar{A}_{\mathrm{r} 2}, \bar{A}_{\mathrm{r} 3}, \bar{A}_{\mathrm{r} 4}\right)$ with $\bar{A}_{\mathrm{rt}} \vdash\left(\left[A_{\mathrm{rt} 2}, A_{\mathrm{rt} 3}\right],\left[A_{\mathrm{rt} 1}, A_{\mathrm{rt} 4}\right]\right), 0 \leq A_{\mathrm{rt} 1} \leq$ $A_{\mathrm{rt} 2}<A_{\mathrm{rt} 3} \leq A_{\mathrm{rt} 4} \cdot \tilde{\bar{I}}_{\mathrm{cs}}=\left(\bar{I}_{\mathrm{cs} 1}, \bar{I}_{\mathrm{cs} 2}, \bar{I}_{\mathrm{cs} 3}, \bar{I}_{\mathrm{cs} 4}\right)$ with $\bar{I}_{\mathrm{cst}} \vdash\left(\left[I_{\mathrm{cst} 2}, I_{\mathrm{cst} 3}\right],\left[I_{\mathrm{cst} 1}, I_{\mathrm{cst} 4}\right]\right)$, $0 \leq I_{\mathrm{cst} 1} \leq I_{\mathrm{cst} 2}<I_{\mathrm{cst} 3} \leq I_{\mathrm{cst} 4} \cdot \tilde{\bar{I}}_{\mathrm{cm}}=\left(\bar{I}_{\mathrm{cm} 1}, \bar{I}_{\mathrm{cm} 2}, \bar{I}_{\mathrm{cm} 3}, \bar{I}_{\mathrm{cm} 4}\right)$ with $\bar{I}_{\mathrm{cmt}} \vdash$ $\left(\left[I_{\mathrm{cmt} 2}, I_{\mathrm{cst} 3}\right],\left[I_{\mathrm{cmt} 1}, I_{\mathrm{cmt} 4}\right]\right), 0 \leq I_{\mathrm{cmt} 1} \leq I_{\mathrm{cmt} 2}<I_{\mathrm{cmt} 3} \leq I_{\mathrm{cmt} 4} \cdot \tilde{\bar{c}}_{\mathrm{tp}}=\left(\bar{c}_{\mathrm{tp} 1}, \bar{c}_{\mathrm{tp} 2}, \bar{c}_{\mathrm{tp} 3}, \bar{c}_{\mathrm{tp} 4}\right)$ with $\bar{c}_{\text {tpt }} \vdash\left(\left[c_{\text {tpt } 2}, c_{\text {tpt } 3}\right],\left[c_{\text {tpt } 1}, c_{\text {tpt } 4}\right]\right), 0 \leq c_{\text {tpt } 1} \leq c_{\text {tpt2 }}<c_{\text {tpt } 3} \leq c_{\text {tpt } 4 \cdot} \cdot \tilde{\overline{c_{\text {tp }}^{\prime}}}=$ $\left({\overline{c^{\prime}}}_{\text {tp1 }},{\overline{c^{\prime}}}_{\text {tp2 }}, \bar{c}_{\text {tp } 3},{\overline{c^{\prime}}}_{\text {tp } 4}\right)$ with ${\overline{c^{\prime}}}_{\text {tpt }} \vdash\left(\left[c_{\text {tpt2 }}^{\prime}, c_{\text {tpt } 3}^{\prime}\right],\left[c_{\text {tpt } 1}^{\prime}, c_{\text {tpt } 4}^{\prime}\right]\right), 0 \leq c_{\text {tpt } 1}^{\prime} \leq c_{\text {tpt } 2}^{\prime}<c_{\text {tpt } 3}^{\prime} \leq$ $c_{\text {tpt } 4}^{\prime}$ 


\section{In equivalent crisp environment}

In this environment, using Lemma 1 and Theorems 1 and 2 , the fuzzy rough objective functions for supplier, manufacturer, and retailer are given by

$$
\begin{aligned}
\text { EAPS }= & E[\mathrm{~A} \tilde{\bar{P}}]=\frac{1}{T}\left[-E\left[\tilde{\bar{Z}}_{0 s}\right]+E\left[\tilde{\bar{Z}}_{1 s}\right] R+E\left[\tilde{\bar{Z}}_{2 s}\right] \frac{R}{P}-E\left[\tilde{\bar{Z}}_{3 s}\right] \frac{R^{2}}{2 P}-E\left[\tilde{\bar{Z}}_{4 s}\right] R^{2}\right] \\
\mathrm{EAPM}= & E[\mathrm{~A} \tilde{\bar{P}} \mathrm{M}] \\
= & \frac{1}{T}\left[-E\left[\tilde{\bar{Z}}_{0 m}\right]-E\left[\tilde{\bar{Z}}_{1 m}\right] \frac{R}{P}+E\left[\tilde{\bar{Z}}_{2 m}\right] R+E\left[\tilde{\bar{Z}}_{3 m}\right] \frac{R^{2}}{P^{2}}+E\left[\tilde{\bar{Z}}_{4 m}\right] \frac{R^{2}}{P}\right. \\
& \left.+E\left[\tilde{\bar{Z}}_{5 m}\right] R^{2}-E\left[\tilde{\bar{Z}}_{6 m}\right] P R^{2}\right] \\
\text { and EAPR }= & E\left[\mathrm{~A} \tilde{\bar{P}}_{R}\right]=\frac{1}{T}\left[E\left[\tilde{\bar{Z}}_{0 r}\right]+E\left[\tilde{\bar{Z}}_{1 r}\right] R+E\left[\tilde{\bar{Z}}_{2 r}\right] \frac{R^{2}}{2 P}+E\left[\tilde{\bar{Z}}_{3 r}\right] R^{2}+E\left[\tilde{\bar{Z}}_{4 r}\right] \frac{R^{3}}{P}\right. \\
& \left.+E\left[\tilde{\bar{Z}}_{5 r}\right] \frac{R^{4}}{P^{2}}\right]
\end{aligned}
$$

Also, the objective functions for integrated model is given by

$$
\begin{aligned}
\mathrm{EIAP}= & E[\mathrm{IA} \overline{\overline{\mathrm{P}}}]=[E[\mathrm{~A} \tilde{\bar{P}}]+E[\mathrm{~A} \tilde{\bar{P}} \mathrm{M}]+E[\mathrm{~A} \tilde{\overline{\mathrm{P}}}]] \\
= & \frac{1}{T}\left[E\left[\tilde{\bar{Z}}_{0}\right]+E\left[\tilde{\bar{Z}}_{1}\right] \frac{R}{P}+E\left[\tilde{\bar{Z}}_{2}\right] R-E\left[\tilde{\bar{Z}}_{3}\right] \frac{R^{2}}{2 P}+\tilde{\bar{Z}}_{4} \frac{R^{2}}{P^{2}}-E\left[\tilde{\bar{Z}}_{5}\right] P R^{2}\right. \\
& \left.-\tilde{\bar{Z}}_{6} R^{2}+E\left[\tilde{\bar{Z}}_{7}\right] \frac{R^{3}}{P}+E\left[\tilde{\bar{Z}}_{8}\right] \frac{R^{4}}{P^{2}}\right]
\end{aligned}
$$

where

$$
\begin{aligned}
& E\left[\tilde{\bar{h}}_{\mathrm{s}}\right]=\frac{1}{16} \sum_{t=1}^{4} \sum_{k=1}^{4} h_{\mathrm{stk}}, \quad E\left[\tilde{\bar{h}}_{\mathrm{m}}\right]=\frac{1}{16} \sum_{t=1}^{4} \sum_{k=1}^{4} h_{\mathrm{mtk}}, \quad E\left[\tilde{\bar{h}}_{\mathrm{m}}^{\prime}\right]=\frac{1}{16} \sum_{t=1}^{4} \sum_{k=1}^{4} h_{\mathrm{mtk}}^{\prime}, \\
& E\left[\tilde{\bar{h}}_{\mathrm{r}}\right]=\frac{1}{16} \sum_{t=1}^{4} \sum_{k=1}^{4} h_{\mathrm{rtk}}, \quad E\left[\tilde{\bar{h}}_{\mathrm{r}}^{\prime}\right]=\frac{1}{16} \sum_{t=1}^{4} \sum_{k=1}^{4} h_{\mathrm{rtk}}^{\prime}, \quad E\left[\tilde{\bar{h}}_{\mathrm{rs}}\right]=\frac{1}{16} \sum_{t=1}^{4} \sum_{k=1}^{4} h_{\mathrm{rstk}}, \\
& E\left[\tilde{\bar{A}}_{\mathrm{s}}\right]=\frac{1}{16} \sum_{t=1}^{4} \sum_{k=1}^{4} A_{\mathrm{stk}}, \quad E\left[\tilde{\bar{A}}_{\mathrm{m}}\right]=\frac{1}{16} \sum_{t=1}^{4} \sum_{k=1}^{4} A_{\mathrm{mtk}}, \quad E\left[\tilde{\bar{A}}_{\mathrm{r}}\right]=\frac{1}{16} \sum_{t=1}^{4} \sum_{k=1}^{4} A_{\mathrm{rtk}} \text {, } \\
& E\left[\tilde{\bar{I}}_{\mathrm{cs}}\right]=\frac{1}{16} \sum_{t=1}^{4} \sum_{k=1}^{4} I_{\mathrm{cstk}}, \quad E\left[\tilde{\bar{I}}_{\mathrm{cm}}\right]=\frac{1}{16} \sum_{t=1}^{4} \sum_{k=1}^{4} I_{\mathrm{cmtk}}, \quad E\left[\tilde{\bar{c}}_{\mathrm{tp}}\right]=\frac{1}{16} \sum_{t=1}^{4} \sum_{k=1}^{4} c_{\mathrm{tptk}}, \\
& E\left[\overline{\tilde{c}_{\text {tp }}^{\prime}}\right]=\frac{1}{16} \sum_{t=1}^{4} \sum_{k=1}^{4} c_{\text {tptk }}^{\prime} .
\end{aligned}
$$

\section{Stakelberg approach (leader-follower relationship)}

In this case, the manufacturer is the leader, and the supplier and retailer are the followers. Also, the optimum values of the average profit of supplier and retailer are obtained by putting the optimum value of the decision variables, which are obtained by optimizing the average profit of manufacturer. Using Equations $6,12 \mathrm{a}, 12 \mathrm{~b}$, and $\left(1-e^{-\alpha t_{1}}\right) \approx \alpha t_{1}$, in Equation 12c, the relation $T=\frac{1-\theta}{D_{\mathrm{r}}} R+t_{0}$ is obtained where $t_{0}$ is given by $t_{0}=\frac{S-W_{1}}{\alpha_{1}+\beta_{1} W_{1}}+$ $\frac{1}{\beta_{1}} \log \left[\left(1-\frac{D_{\mathrm{r}}}{\alpha_{1}}\right)\left(1-e^{-\beta_{1} t_{3}}\right)\right]$ and it is independent of $R$ and $P$. 


\section{When both $R$ and $P$ are decision variables}

The average profit of manufacturer is given by

$$
\begin{aligned}
\operatorname{EAPM}(R, P)= & \frac{1}{T}\left[-E\left[\tilde{\bar{Z}}_{0 m}\right]-E\left[\tilde{\bar{Z}}_{1 m}\right] \frac{R}{P}+E\left[\tilde{\bar{Z}}_{2 m}\right] R+E\left[\tilde{\bar{Z}}_{3 m}\right] \frac{R^{2}}{P^{2}}+E\left[\tilde{\bar{Z}}_{4 m}\right] \frac{R^{2}}{P}\right. \\
& \left.+E\left[\tilde{\bar{Z}}_{5 m}\right] R^{2}-E\left[\tilde{\bar{Z}}_{6 m}\right] P R^{2}\right]
\end{aligned}
$$

where $E\left[\tilde{\bar{Z}}_{i m}\right], i=0,1,2, \ldots, 6$, are independent of $R$ and $P$. (See Appendix 1 ).

The necessary conditions for maximum value of $\operatorname{EAPM}(R, P)$ are $\frac{\partial}{\partial R}(\mathrm{EAPM})=0$ and $\frac{\partial}{\partial P}(\mathrm{EAPM})=0$.

Now, $\frac{\partial}{\partial R}(\mathrm{EAPM})=0$

$$
\text { i.e., } \begin{aligned}
\frac{(1-\theta)}{D_{\mathrm{r}} T} E\left[\tilde{\bar{Z}}_{0 m}\right] & +\left\{\frac{(1-\theta) R}{D_{\mathrm{r}} P T}-\frac{1}{P}\right\} E\left[\tilde{\bar{Z}}_{1 m}\right]+\left\{1-\frac{(1-\theta) R}{D_{\mathrm{r}} T}\right\} E\left[\tilde{\bar{Z}}_{2 m}\right] \\
& +\left\{\frac{2 R}{P^{2}}-\frac{(1-\theta) R^{2}}{D_{\mathrm{r}} T P^{2}}\right\} E\left[\tilde{\bar{Z}}_{3 m}\right]+\left\{\frac{2 R}{P}-\frac{(1-\theta) R^{2}}{D_{\mathrm{r}} T P}\right\} E\left[\tilde{\bar{Z}}_{4 m}\right] \\
& +\left\{2 R-\frac{(1-\theta) R^{2}}{D_{\mathrm{r}} T}\right\} E\left[\tilde{\bar{Z}}_{5 m}\right]+\left\{\frac{(1-\theta) R^{2} P}{D_{\mathrm{r}} T}-2 P R\right\} E\left[\tilde{\bar{Z}}_{6 m}\right]=0
\end{aligned}
$$

and $\frac{\partial}{\partial P}(\mathrm{EAPM})=0$

$$
\text { i.e., } E\left[\tilde{\bar{Z}}_{1 m}\right] \frac{R}{P^{2}}-2 E\left[\tilde{\bar{Z}}_{3 m}\right] \frac{R^{2}}{P^{3}}-E\left[\tilde{\bar{Z}}_{4 m}\right] \frac{R^{2}}{P^{2}}-E\left[\tilde{\bar{Z}}_{6 m}\right] R^{2}=0
$$

Solving (14) and (15), we can obtain the optimum value of $R$ and $P$, say $R^{*}$ and $P^{*}$.

If $\frac{\partial^{2}}{\partial R^{2}}(\mathrm{EAPM})<0, \frac{\partial^{2}}{\partial P^{2}}(\mathrm{EAPM})<0$ and $\left\{\frac{\partial^{2}}{\partial R^{2}}(\mathrm{EAPM})\right\}\left\{\frac{\partial^{2}}{\partial P^{2}}(\mathrm{EAPM})\right\}-$ $\left\{\frac{\partial^{2}}{\partial P \partial R}(\mathrm{EAPM})\right\}^{2}>0$ holds for $R=R^{*}$ and $P=P^{*}$, then EAPM $\left(R^{*}, P^{*}\right)$ is maximum.

Now, $\left.\frac{\partial^{2}}{\partial R^{2}}(\mathrm{EAPM})\right]_{\text {at }\left(R^{*}, P^{*}\right)}<0$

$$
\begin{aligned}
\text { i.e., } & -\frac{(1-\theta)^{2}}{D_{\mathrm{r}}^{2} T^{* 2}} E\left[\tilde{\bar{Z}}_{0 m}\right]+\left\{\frac{(1-\theta)}{D_{\mathrm{r}} T^{*} P^{*}}-\frac{(1-\theta)^{2} R^{*}}{D_{\mathrm{r}}{ }^{2} T^{* 2} P^{*}}\right\} E\left[\tilde{\bar{Z}}_{1 m}\right] \\
& +\left\{\frac{(1-\theta)^{2} R^{*}}{D_{\mathrm{r}}^{2} T^{* 2}}-\frac{(1-\theta)}{D_{\mathrm{r}} T^{*}}\right\} E\left[\tilde{\bar{Z}}_{2 m}\right]+\left\{\frac{(1-\theta)^{2} R^{* 2}}{D_{\mathrm{r}}{ }^{2} T^{* 2} P^{* 2}}+\frac{1}{P^{* 2}}-\frac{2(1-\theta) R^{*}}{D_{\mathrm{r}} T^{*} P^{* 2}}\right\} E\left[\tilde{\bar{Z}}_{3 m}\right] \\
& +\left\{\frac{(1-\theta)^{2}}{D_{\mathrm{r}}^{2} T^{* 2} P^{*}}+\frac{1}{P^{*}}-\frac{2(1-\theta) R^{*}}{D_{\mathrm{r}} T^{*} P^{*}}\right\} E\left[\tilde{\bar{Z}}_{4 m}\right]+\left\{1-\frac{2(1-\theta) R^{*}}{D_{\mathrm{r}} T^{*} P^{*}}+\frac{(1-\theta)^{2} R^{* 2}}{D_{\mathrm{r}}{ }^{2} T^{* 2}}\right\} E\left[\tilde{\bar{Z}}_{5 m}\right] \\
& -\left\{P^{*}-\frac{2(1-\theta) P^{*} R^{*}}{D_{\mathrm{r}} T^{*}}+\frac{(1-\theta)^{2} R^{* 2} P^{*}}{D_{\mathrm{r}}{ }^{2} T^{* 2}}\right\} E\left[\tilde{\bar{Z}}_{6 m}\right]<0
\end{aligned}
$$

and $\left.\frac{\partial^{2}}{\partial P^{2}}(\mathrm{EAPM})\right]_{\mathrm{at}}\left(R^{*}, P^{*}\right)<0$

$$
\begin{aligned}
& \text { i.e., }-E\left[\tilde{\bar{Z}}_{1 m}\right] \frac{R^{*}}{P^{* 3}}+3 E\left[\tilde{\bar{Z}}_{3 m}\right] \frac{R^{* 2}}{P^{* 4}}+E\left[\tilde{\bar{Z}}_{4 m}\right] \frac{R^{* 2}}{P^{* 3}}<0 \\
& \text { and }\left[\left\{\frac{\partial^{2}}{\partial R^{2}}(\mathrm{EAPM})\right\}\left\{\frac{\partial^{2}}{\partial P^{2}}(\mathrm{EAPM})\right\}-\left\{\frac{\partial^{2}}{\partial P \partial R}(\mathrm{EAPM})\right\}^{2}\right]_{\text {at }\left(R^{*}, P^{*}\right)}>0
\end{aligned}
$$

Therefore, EAPM $\left(R^{*}, P^{*}\right)$ is maximum if the relations (16), (17), and (18) hold. 
The corresponding optimum average profit of supplier and retailer is

$$
\begin{aligned}
\operatorname{EAPS}\left(R^{*}, P^{*}\right)=\frac{1}{T^{*}} & {\left[-E\left[\tilde{\bar{Z}}_{0 s}\right]+E\left[\tilde{\bar{Z}}_{1 s}\right] R^{*}+E\left[\tilde{\bar{Z}}_{2 s}\right] \frac{R^{*}}{P^{*}}-E\left[\tilde{\bar{Z}}_{3 s}\right] \frac{R^{* 2}}{2 P^{*}}-E\left[\tilde{\bar{Z}}_{4 s}\right] R^{* 2}\right] } \\
\operatorname{EAPR}\left(R^{*}, P^{*}\right)=\frac{1}{T^{*}} & {\left[E\left[\tilde{\bar{Z}}_{0 r}\right]+E\left[\tilde { \overline { Z } } _ { 1 r } \left[R^{*}+E\left[\tilde{\bar{Z}}_{2 r}\right] \frac{R^{* 2}}{2 P^{*}}+E\left[\tilde{\bar{Z}}_{3 r}\right] R^{* 2}+E\left[\tilde{\bar{Z}}_{4 r}\right] \frac{R^{* 3}}{P^{*}}\right.\right.\right.} \\
& \left.+E\left[\tilde{\bar{Z}}_{5 r}\right] \frac{R^{* 4}}{P^{* 2}}\right]
\end{aligned}
$$

where $T^{*}=\frac{1-\theta}{D_{\mathrm{r}}} R^{*}+t_{0}$.

\section{When $P$ is a decision variable}

The necessary conditions for maximum value of $\operatorname{EAPM}(P)$ is $\frac{d}{d P}(\mathrm{EAPM})=0$

$$
\text { i.e., } E\left[\tilde{\bar{Z}}_{1 m}\right] \frac{R}{P^{2}}-2 E\left[\tilde{\bar{Z}}_{3 m}\right] \frac{R^{2}}{P^{3}}-E\left[\tilde{\bar{Z}}_{4 m}\right] \frac{R^{2}}{P^{2}}-E\left[\tilde{\bar{Z}}_{6 m}\right] R^{2}=0
$$

which gives the optimum value of $P$, say $P^{* *}$. (See Appendix 2).

If $\frac{d^{2}}{d P^{2}}(\mathrm{EAPM})<0$ holds for $P=P^{* *}$, then EAPM $\left(P^{* *}\right)$ is maximum.

$$
\text { Now } \left.\frac{d^{2}}{d P^{2}}(\text { EAPM })\right]_{\text {at } P=P^{* *}}<0 \text { gives }-E\left[\tilde{\bar{Z}}_{1 m}\right] \frac{R}{P^{* * 3}}+3 E\left[\tilde{\bar{Z}}_{3 m}\right] \frac{R^{2}}{P^{* * 4}}+E\left[\tilde{\bar{Z}}_{4 m}\right] \frac{R^{2}}{P^{* * 3}}<0
$$

Therefore, $\operatorname{EAPM}\left(P^{* *}\right)$ is maximum if the relation $(18 \mathrm{~b})$ holds and the corresponding optimum average profits of supplier and retailer are respectively

$$
\begin{aligned}
\operatorname{EAPS}\left(P^{* *}\right)=\frac{1}{T} & {\left[-E\left[\tilde{\bar{Z}}_{0 s}\right]+E\left[\tilde{\bar{Z}}_{1 s}\right] R+E\left[\tilde{\bar{Z}}_{2 s}\right] \frac{R}{P^{* *}}-E\left[\tilde{\bar{Z}}_{3 s}\right] \frac{R^{2}}{2 P^{* *}}-E\left[\tilde{\bar{Z}}_{4 s}\right] R^{2}\right] } \\
\operatorname{EAPR}\left(P^{* *}\right)=\frac{1}{T} & {\left[E\left[\tilde{\bar{Z}}_{0 r}\right]+E\left[\tilde{\bar{Z}}_{1 r}\right] R+E\left[\tilde{\bar{Z}}_{2 r}\right] \frac{R^{2}}{2 P^{* *}}+E\left[\tilde{\bar{Z}}_{3 r}\right] R^{2}+E\left[\tilde{\bar{Z}}_{4 r}\right] \frac{R^{3}}{P^{* *}}\right.} \\
& \left.+E\left[\tilde{\bar{Z}}_{5 r}\right] \frac{R^{4}}{P^{* * 2}}\right]
\end{aligned}
$$

where $T=\frac{1-\theta}{D_{\mathrm{r}}} R+t_{0}$.

\section{Integrated approach}

\section{When both $R$ and $P$ are decision variables}

The necessary conditions for maximum value of $\operatorname{EIAP}(R, P)$ are $\frac{\partial}{\partial R}(\operatorname{EIAP})=0$ and $\frac{\partial}{\partial P}($ EIAP $)=0$

Now, $\frac{\partial}{\partial R}($ EIAP $)=0$

$$
\begin{aligned}
\text { i.e., } & -\frac{(1-\theta)}{D_{\mathrm{r}} T} E\left[\tilde{\bar{Z}}_{0}\right]+\left\{\frac{1}{P}-\frac{(1-\theta) R}{D_{\mathrm{r}} T P}\right\} E\left[\tilde{\bar{Z}}_{1}\right]+\left\{1-\frac{(1-\theta) R}{D_{\mathrm{r}} T}\right\} E\left[\tilde{\bar{Z}}_{2}\right]-\left\{\frac{R}{P}+\frac{(1-\theta) R^{2}}{2 D_{\mathrm{r}} T P}\right\} \\
& \times E\left[\tilde{\bar{Z}}_{3}\right]+\left\{\frac{2 R}{P^{2}}-\frac{(1-\theta) R^{2}}{D_{\mathrm{r}} T P^{2}}\right\} E\left[\tilde{\bar{Z}}_{4}\right]-\left\{2 P R+\frac{(1-\theta) P R^{2}}{D_{\mathrm{r}} T}\right\} E\left[\tilde{\bar{Z}}_{5}\right]-\left\{2 R+\frac{(1-\theta) R^{2}}{D_{\mathrm{r}} T}\right\} \\
& \times E\left[\tilde{\bar{Z}}_{6}\right]+\left\{\frac{3 R^{2}}{P}-\frac{(1-\theta) R^{3}}{D_{\mathrm{r}} T P}\right\} E\left[\tilde{\bar{Z}}_{7}\right]+\left\{\frac{4 R^{3}}{P^{2}}-\frac{(1-\theta) R^{4}}{D_{\mathrm{r}} T P^{2}}\right\} E\left[\tilde{\bar{Z}}_{8}\right]=0
\end{aligned}
$$

and $\frac{\partial}{\partial P}(\mathrm{EIAP})=0$

$$
\text { i.e., }\left[-E\left[\tilde{\bar{Z}}_{1}\right] \frac{R}{P^{2}}-E\left[\tilde{\bar{Z}}_{3}\right] \frac{R^{2}}{2 P^{2}}-2 E\left[\tilde{\bar{Z}}_{4}\right] \frac{R^{2}}{P^{3}}-E\left[\tilde{\bar{Z}}_{5}\right] R^{2}-E\left[\tilde{\bar{Z}}_{7}\right] \frac{R^{3}}{P^{2}}-2 E\left[\tilde{\bar{Z}}_{8}\right] \frac{R^{4}}{P^{3}}\right]=0(20)
$$

Solving (19) and (20), we can obtain the optimum value of $R$ and $P$, say $R^{*}$ and $P^{*}$. 
Table 1 Collected data for different crisp parameters

\begin{tabular}{lclclc}
\hline Parameters & Value & Parameter & Value & Parameter & Value \\
\hline$\theta$ & 0.11 & $C_{s}$ & 40 & $S$ & 335 \\
$x$ & 20 & $W_{s}$ & 90 & $W_{1}$ & 45 \\
$\alpha$ & 0.10 & $W_{s}^{\prime}$ & 55 & $W_{2}$ & 16 \\
$\beta$ & 0.44 & $R_{1}$ & 16 & $a$ & 51 \\
$\gamma$ & 0.78 & $H$ & 0.02 & $b$ & 0.17 \\
$\alpha_{1}$ & 20 & $G$ & 10 & $D_{r}^{\prime}$ & 35 \\
$\beta_{1}$ & 0.2 & $S_{r}$ & 176 & $D_{r}$ & 36 \\
$r_{c m}$ & 2 & $S_{r}^{\prime}$ & 135 & $S_{c}$ & 0.35 \\
$S_{m}$ & 158 & $S_{m}^{\prime}$ & 108 & & \\
\hline
\end{tabular}

If $\frac{\partial^{2}}{\partial R^{2}}(\mathrm{EIAP})<0, \frac{\partial^{2}}{\partial P^{2}}(\mathrm{EIAP})<0$ and $\left\{\frac{\partial^{2}}{\partial R^{2}}(\mathrm{EIAP})\right\}\left\{\frac{\partial^{2}}{\partial P^{2}}(\mathrm{EIAP})\right\}-\left\{\frac{\partial^{2}}{\partial P \partial R}(\mathrm{EIAP})\right\}^{2}>0$ holds for $R=R^{*}$ and $P=P^{*}$ then $\operatorname{EIAP}\left(R^{*}, P^{*}\right)$ is maximum.

Now, $\frac{\partial^{2}}{\partial R^{2}}(\text { EIAP) }]_{\text {at }\left(R^{*}, P^{*}\right)}<0$

$$
\begin{aligned}
& \text { i.e., } \frac{2(1-\theta)^{2}}{D_{\mathrm{r}}^{2} T^{* 2}} E\left[\tilde{\bar{Z}}_{0}\right]+\left\{\frac{2(1-\theta)^{2} R^{*}}{D_{\mathrm{r}}^{2} T^{* 2} P^{*}}-\frac{2(1-\theta)}{D_{\mathrm{r} T^{*} P^{*}}}\right\} E\left[\tilde{\bar{Z}}_{1}\right]+\left\{\frac{2(1-\theta)^{2} R^{*}}{D_{\mathrm{r}}^{2} T^{* 2}}-\frac{2(1-\theta)}{D_{\mathrm{r} T^{*}}}\right\} E\left[\tilde{\bar{Z}}_{2}\right] \\
& -\left\{1+\frac{2(1-\theta)^{2} R^{* 2}}{D_{\mathrm{r}}^{2} T^{* 2} P^{*}}-\frac{2(1-\theta) R^{*}}{D_{\mathrm{r} T^{*}} P^{*}}\right\} E\left[\tilde{\bar{Z}}_{3}\right]+\left\{\frac{2}{P^{* 2}}+\frac{2(1-\theta)^{2} R^{* 2}}{D_{\mathrm{r}}^{2} T^{*}} P^{* 2}-\frac{4(1-\theta) R^{*}}{D_{\mathrm{r} T^{*}} P^{*}}\right\} E\left[\tilde{\bar{Z}}_{4}\right] \\
& -\left\{2 P^{*}+\frac{2(1-\theta)^{2} P^{*} R^{* 2}}{D_{\mathrm{r}}^{2} T^{* 2}}-\frac{4(1-\theta) P^{*} R^{*}}{D_{\mathrm{r} T^{*}}}\right\} E\left[\tilde{\bar{Z}}_{5}\right]-\left\{2+\frac{2(1-\theta)^{2} R^{* 2}}{D_{\mathrm{r}}^{2} T^{* 2}}-\frac{4(1-\theta) R^{*}}{D_{\mathrm{r} T}}\right\} E\left[\tilde{\bar{Z}}_{6}\right] \\
& +\left\{\frac{6 R^{*}}{P^{*}}+\frac{2(1-\theta)^{2} R^{* 3}}{D_{\mathrm{r}}^{2} T^{* 2} P^{*}}-\frac{6(1-\theta) R^{* 2}}{D_{\mathrm{r} T^{*} P^{*}}}\right\} E\left[\tilde{\bar{Z}}_{7}\right]+\left\{\frac{12 R^{* 2}}{P^{* 2}}+\frac{2(1-\theta)^{2} R^{* 4}}{D_{\mathrm{r}}^{2} T^{* 2} P^{* 2}}-\frac{8(1-\theta) R^{* 3}}{D_{\mathrm{r} T^{*} P^{*}}^{2}}\right\} E\left[\tilde{\bar{Z}}_{8}\right]<0
\end{aligned}
$$

\begin{tabular}{|c|c|c|c|c|}
\hline $\begin{array}{l}\text { Fu-Ro } \\
\text { parameters }\end{array}$ & $\begin{array}{l}\text { Fu-Ro } \\
\text { value }\end{array}$ & $\begin{array}{l}\text { Input } \\
\text { values }\end{array}$ & $\begin{array}{l}\text { Expected } \\
\text { value }\end{array}$ & Value \\
\hline$\tilde{\bar{h}}_{\mathrm{s}}$ & Near roughly $(0.5)$ & $(\overline{0.49}, \overline{0.50}, \overline{0.51}, \overline{0.52})$ with $([-0.04,0.04],[-0.08,0.08])$ & $E\left[\tilde{\bar{h}}_{\mathrm{s}}\right]$ & 5.05 \\
\hline$\tilde{\bar{h}}_{\mathrm{m}}$ & Near roughly $(1.7)$ & $(\overline{1.5}, \overline{1.6}, \overline{1.7}, \overline{1.8})$ with $([-0.2,0.2],[-0.4,0.4])$ & $E\left[\tilde{\bar{h}}_{m}\right]$ & 1.65 \\
\hline$\tilde{\bar{h}}_{\mathrm{m}}^{\prime}$ & Near roughly (1.5) & $(\overline{1.35}, \overline{1.40}, \overline{1.50}, \overline{1.55})$ with $([-0.18,0.18],[-0.36,0.36])$ & $E\left[\tilde{\overline{h_{m}^{\prime}}}\right]$ & 1.45 \\
\hline$\tilde{\bar{h}}_{r}$ & Near roughly (1.6) & $(\overline{1.54}, \overline{1.6}, \overline{1.63}, \overline{1.67})$ with $([-0.12,0.12],[-0.24,0.24])$ & $E\left[\tilde{\bar{h}}_{r}\right]$ & 1.61 \\
\hline$\tilde{\overline{h_{r}^{\prime}}}$ & Near roughly (1.3) & $(\overline{1.15}, \overline{1.22}, \overline{1.3}, \overline{1.37})$ with $([-0.11,0.11],[-0.22,0.22])$ & $E\left[\tilde{\overline{h_{r}^{\prime}}}\right]$ & 1.26 \\
\hline$\tilde{\bar{h}}_{\mathrm{rs}}$ & Near roughly (1.8) & $(\overline{1.67}, \overline{1.72}, \overline{1.80}, \overline{1.85})$ with $([-0.13,0.13],[-0.26,0.26])$ & $E\left[\tilde{\bar{h}}_{\mathrm{rs}}\right]$ & 1.78 \\
\hline$\tilde{\bar{A}}_{s}$ & Near roughly (420) & $(\overline{416}, \overline{420}, \overline{425}, \overline{428})$ with $([-0.30,0.30],[-0.60,0.60])$ & $E\left[\tilde{\bar{A}}_{s}\right]$ & 422.25 \\
\hline$\tilde{\bar{A}}_{m}$ & Near roughly (500) & $(\overline{486}, \overline{495}, \overline{500}, \overline{510})$ with $([-0.29,0.29],[-0.58,0.58])$ & $E\left[\tilde{\bar{A}}_{\mathrm{m}}\right]$ & 491.50 \\
\hline$\tilde{\bar{A}}_{r}$ & Near roughly (400) & $(\overline{395}, \overline{400}, \overline{408}, \overline{410})$ with $([-0.35,0.35],[-0.70,0.70])$ & $E\left[\tilde{\bar{A}}_{\mathrm{r}}\right]$ & 403.25 \\
\hline$\tilde{\bar{l}}_{c s}$ & Near roughly (3) & $(\overline{2.5}, \overline{2.8}, \overline{3.0}, \overline{3.15})$ with $([-0.3,0.3],[-0.6,0.6])$ & $E\left[\tilde{\bar{l}}_{\mathrm{cS}}\right]$ & 2.86 \\
\hline$\tilde{\bar{l}}_{\mathrm{cm}}$ & Near roughly (2) & $(\overline{1.9}, \overline{1.96}, \overline{2.0}, \overline{2.04})$ with $([-0.21,0.21],[-0.42,0.42])$ & $E\left[\tilde{\bar{l}}_{\mathrm{cm}}\right]$ & 1.97 \\
\hline$\tilde{\bar{c}}_{\mathrm{tp}}$ & Near roughly (1.4) & $(\overline{1.36}, \overline{1.40}, \overline{1.43}, \overline{1.47})$ with $([-0.14,0.14],[-0.28,0.28])$ & $E\left[\tilde{\bar{c}}_{\mathrm{tp}}\right]$ & 1.42 \\
\hline$\tilde{\widetilde{c_{\mathrm{tp}}^{\prime}}}$ & Near roughly (1.0) & $(\overline{0.90}, \overline{0.97}, \overline{1.0}, \overline{1.06})$ with $([-0.01,0.01],[-0.02,0.02])$ & $\tilde{E}\left[\tilde{\overline{c_{\mathrm{tp}}^{\prime}}}\right]$ & 0.98 \\
\hline
\end{tabular}

Table 2 Collected data for different fuzzy-rough parameters

The value of $\tilde{\bar{h}}_{\mathrm{s}}$ is near roughly $(0.5)=(\overline{0.49}, \overline{0.50}, \overline{0.51}, \overline{52})$ with oscillation $([-0.04,0.04],[-0.08,0.08])$ means that $\overline{0.49} \vdash([0.45,0.53],[0.41,0.57]), \overline{0.50} \vdash([0.46,0.54],[0.42,0.58]), \overline{0.51} \vdash([0.47,0.55],[0.43,0.59])$ and $\overline{0.52} \vdash([0.48,0.56],[0.44,0.60])$. 
Table 3 Optimal result when $P$ and $R$ are decision variables

\begin{tabular}{lcccccc}
\hline Approach & Total profit & EAPS & EAPM & EAPR & $\boldsymbol{R}$ & $\boldsymbol{P}$ \\
\hline Stakelberg & $5,126.616$ & $1,43.341$ & $1,814.635$ & $1,656.160$ & $3,217.589$ & 64.9821 \\
Integrated & $5,176.606$ & $1,596.751$ & $1,872.695$ & $1,707.160$ & $3,189.629$ & 69.8521 \\
\hline
\end{tabular}

and $\left.\frac{\partial^{2}}{\partial P^{2}}(\mathrm{EIAP})\right]_{\mathrm{at}\left(R^{*}, P^{*}\right)}$

$$
\begin{array}{r}
\text { i.e., }\left[2 E\left[\tilde{\bar{Z}}_{1}\right] \frac{R^{*}}{P^{* 3}}+E\left[\tilde{\bar{Z}}_{3}\right] \frac{R^{* 2}}{P^{* 3}}+6 E\left[\tilde{\bar{Z}}_{4}\right] \frac{R^{* 2}}{P^{* 4}}+2 E\left[\tilde{\bar{Z}}_{7}\right] \frac{R^{* 3}}{P^{* 3}}+6 E\left[\tilde{\bar{Z}}_{8}\right] \frac{R^{* 4}}{P^{* 4}}\right]<0 \\
\text { and }\left[\left\{\frac{\partial^{2}}{\partial R^{2}}(\text { EIAP) }\}\left\{\frac{\partial^{2}}{\partial P^{2}}(\text { EIAP) }\}-\left\{\frac{\partial^{2}}{\partial P \partial R}(\text { EIAP })\right\}^{2}\right]_{\mathrm{at}\left(R^{*}, P^{*}\right)}>0\right.\right.
\end{array}
$$

Therefore, $\operatorname{IAP}\left(R^{*}, P^{*}\right)$ is maximum if the relations (21), (22), and (23) hold and the corresponding optimum integrated average profit of the supply chain is

$$
\begin{aligned}
\operatorname{EIAP}\left(R^{*}, P^{*}\right)= & \frac{1}{T^{*}}\left[E\left[\tilde{\bar{Z}}_{0}\right]+E\left[\tilde{\bar{Z}}_{1}\right] \frac{R^{*}}{P^{*}}+E\left[\tilde{\bar{Z}}_{2}\right] R^{*}-Z_{3} \frac{R^{* 2}}{2 P^{*}}+E\left[\tilde{\bar{Z}}_{4}\right] \frac{R^{* 2}}{P^{* 2}}\right. \\
& \left.-E\left[\tilde{\bar{Z}}_{5}\right] P^{*} R^{* 2}-E\left[\tilde{\bar{Z}}_{6}\right] R^{* 2}+E\left[\tilde{\bar{Z}}_{7}\right] \frac{R^{*} 3}{P^{*}}+E\left[\tilde{\bar{Z}}_{8}\right] \frac{R^{* 4}}{P^{* 2}}\right] .
\end{aligned}
$$

where $T^{*}=\frac{1-\theta}{D_{\mathrm{r}}} R^{*}+t_{0}$.

\section{When $P$ is a decision variable}

The necessary conditions for maximum value of $\operatorname{EIAP}(P)$ is $\frac{d}{d P}(\operatorname{EIAP})=0$ i.e., $E\left[\tilde{\bar{Z}}_{1}\right]$ $\frac{R}{P^{2}}-E\left[\tilde{\bar{Z}}_{3}\right] \frac{R^{2}}{2 P^{2}}+2 E\left[\tilde{\bar{Z}}_{4}\right] \frac{R^{2}}{P^{3}}+E\left[\tilde{\bar{Z}}_{5}\right] R^{2}-E\left[\tilde{\bar{Z}}_{7}\right] \frac{R^{3}}{P^{2}}+2 E\left[\tilde{\bar{Z}}_{8}\right] \frac{R^{4}}{P^{3}}=0$ which gives the optimum value of $P$, say $P^{* *}$.

If $\frac{d^{2}}{d P^{2}}$ (EIAP) $<0$ hold for $P=P^{* *}$ then $\operatorname{EIAP}\left(P^{* *}\right)$ is maximum.

Now $\frac{d^{2}}{d P^{2}}(\text { EIAP) }]_{\text {at } P=P^{* *}}<0$ gives

$$
-2 E\left[\tilde{\bar{Z}}_{1}\right] \frac{R}{P^{* * 3}}+E\left[\tilde{\bar{Z}}_{3}\right] \frac{R^{2}}{P^{* * 3}}-6 E\left[\tilde{\bar{Z}}_{4}\right] \frac{R^{2}}{P^{* * 4}}-2 E\left[\tilde{\bar{Z}}_{7}\right] \frac{R^{3}}{P^{* * 3}}-6 E\left[\tilde{\bar{Z}}_{8}\right] \frac{R^{4}}{P^{* * 4}}<0(24)
$$

Therefore, $\operatorname{EIAP}\left(P^{* *}\right)$ is maximum if the relation (24) holds and the corresponding maximum integrated average profit of the supply chain is

$$
\begin{aligned}
\operatorname{EIAP}\left(P^{* *}\right)= & \frac{1}{T}\left[E\left[\tilde{\bar{Z}}_{0}\right]+E\left[\tilde{\bar{Z}}_{1}\right] \frac{R}{P^{* *}}+Z_{2} R-Z_{3} \frac{R^{2}}{2 P^{* *}}+E\left[\tilde{\bar{Z}}_{4}\right] \frac{R^{2}}{P^{* * 2}}\right. \\
& \left.-Z_{5} P_{2} R^{2}-E\left[\tilde{\bar{Z}}_{6}\right] R^{2}+E\left[\tilde{\bar{Z}}_{7}\right] \frac{R^{3}}{P^{* *}}+E\left[\tilde{\bar{Z}}_{8}\right] \frac{R^{4}}{P^{* * 2}}\right] .
\end{aligned}
$$

Table 4 Optimal result when $P$ is decision variable when $R=3,225$

\begin{tabular}{lccccc}
\hline Approach & Total profit & EAPS & EAPM & EAPR & $\boldsymbol{P}$ \\
\hline Stakelberg & $5,012.614$ & $1,512.752$ & $1,714.346$ & $1,751.426$ & 63.64570 \\
Integrated & $5,049.634$ & $1,582.912$ & $1,759.146$ & $1,707.576$ & 67.37870 \\
\hline
\end{tabular}



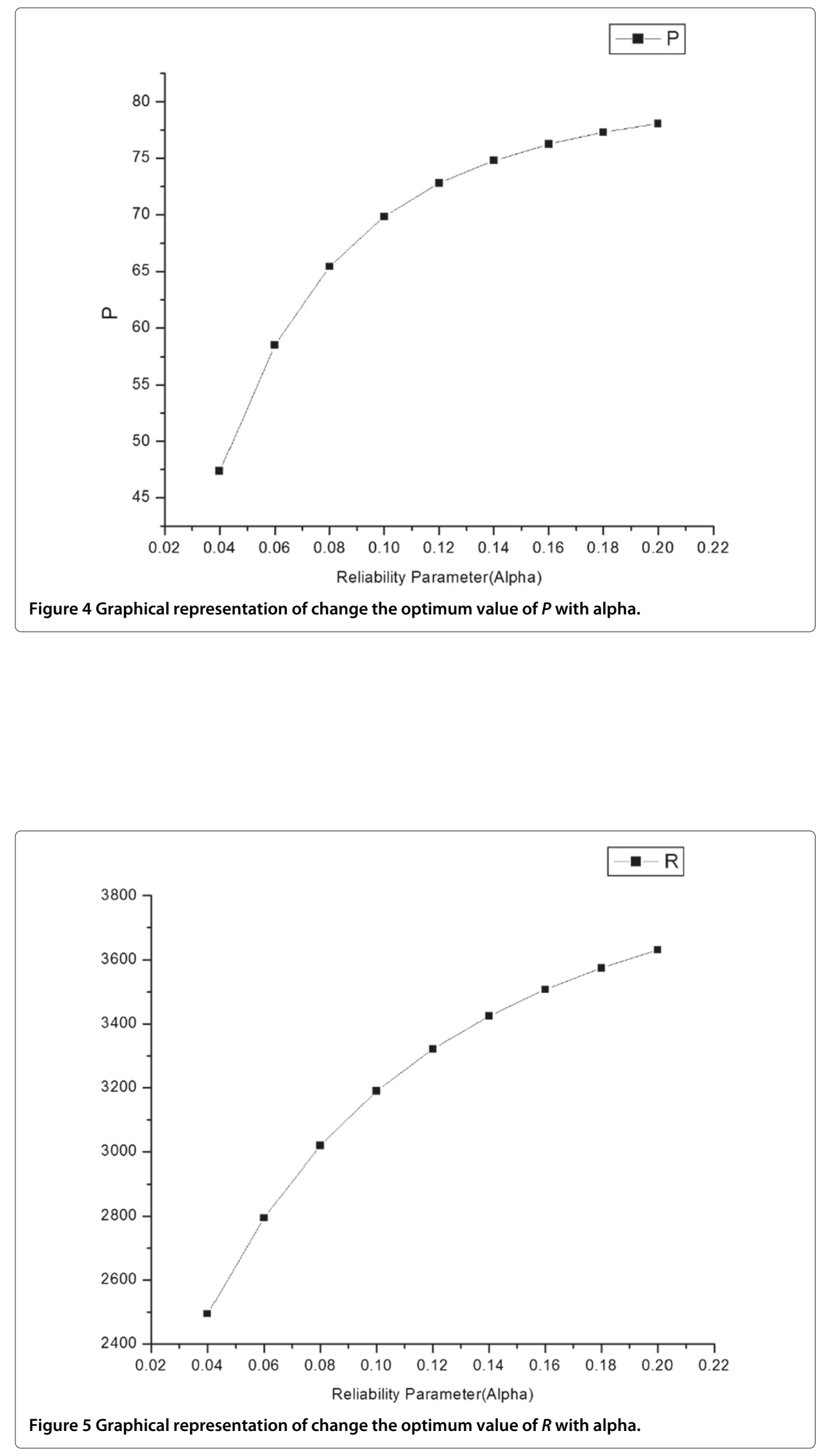


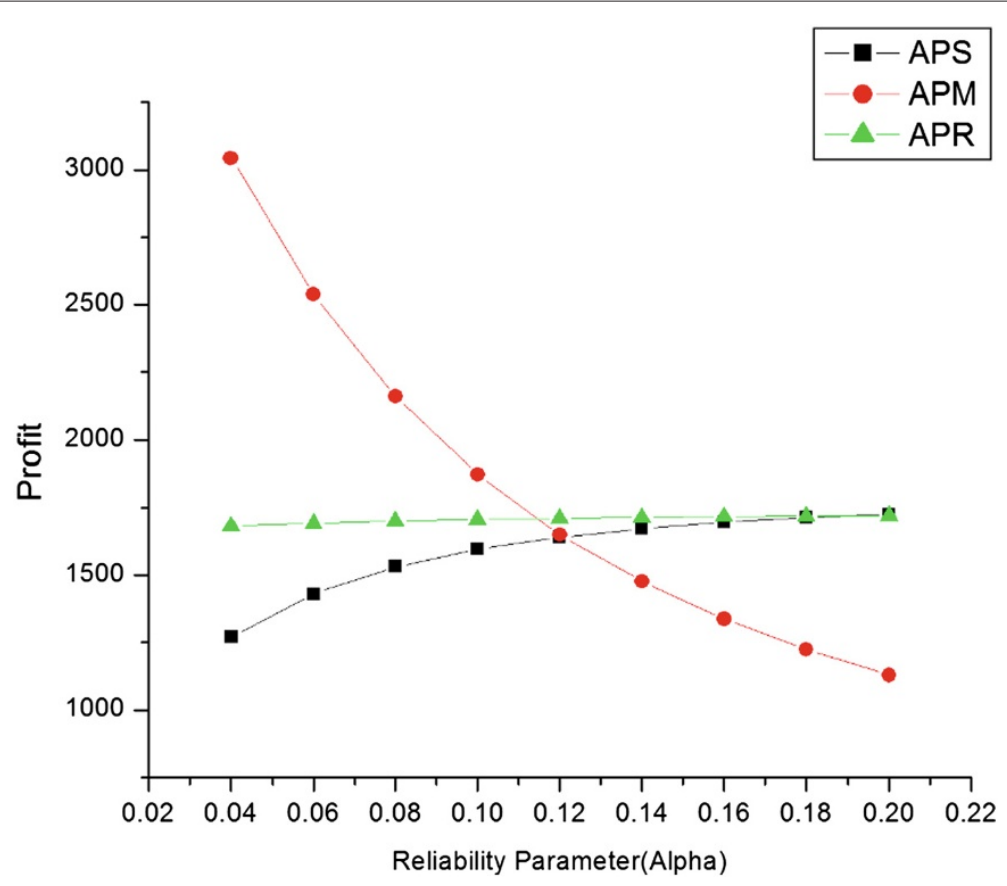

Figure 6 Graphical representation of change the optimum value of profits with alpha.

\section{Numerical examples}

To illustrate the proposed production inventory model, we consider the following numerical data in Tables 1 and 2.

The optimal values of the decision variables and corresponding profits are given in Tables 3 and 4. Also, sensitivity analysis has been performed of the profits, production

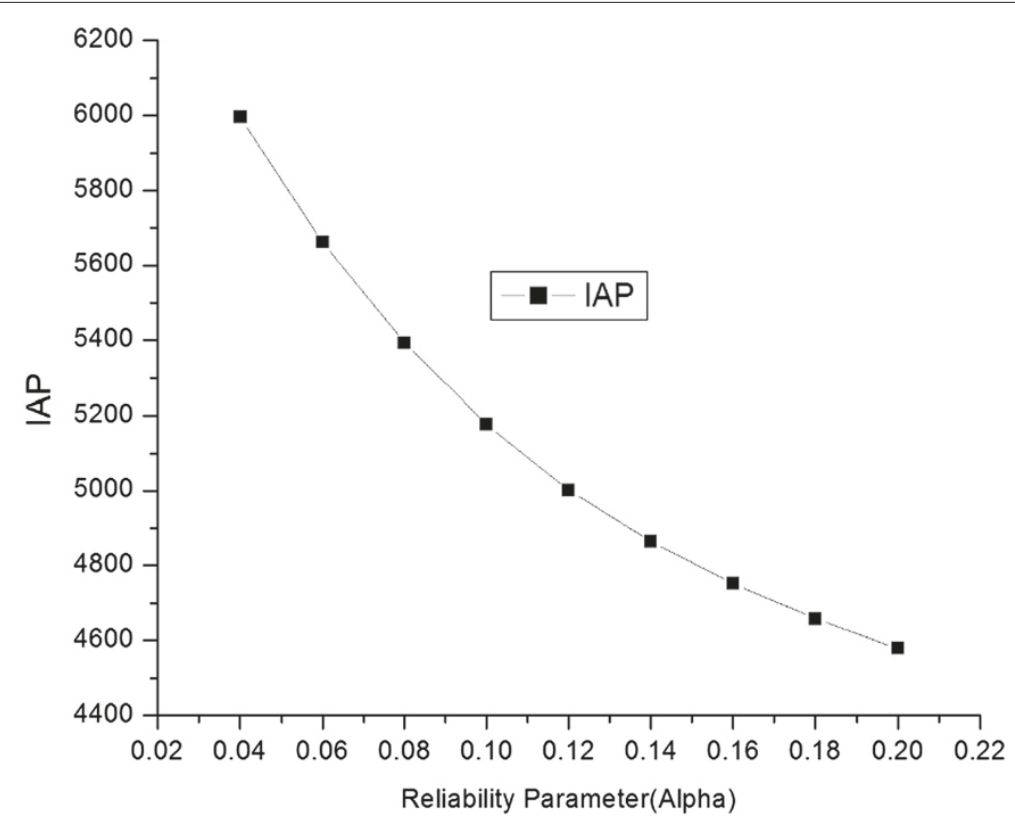

Figure 7 Graphical representation of change the optimum value of IAP with alpha. 


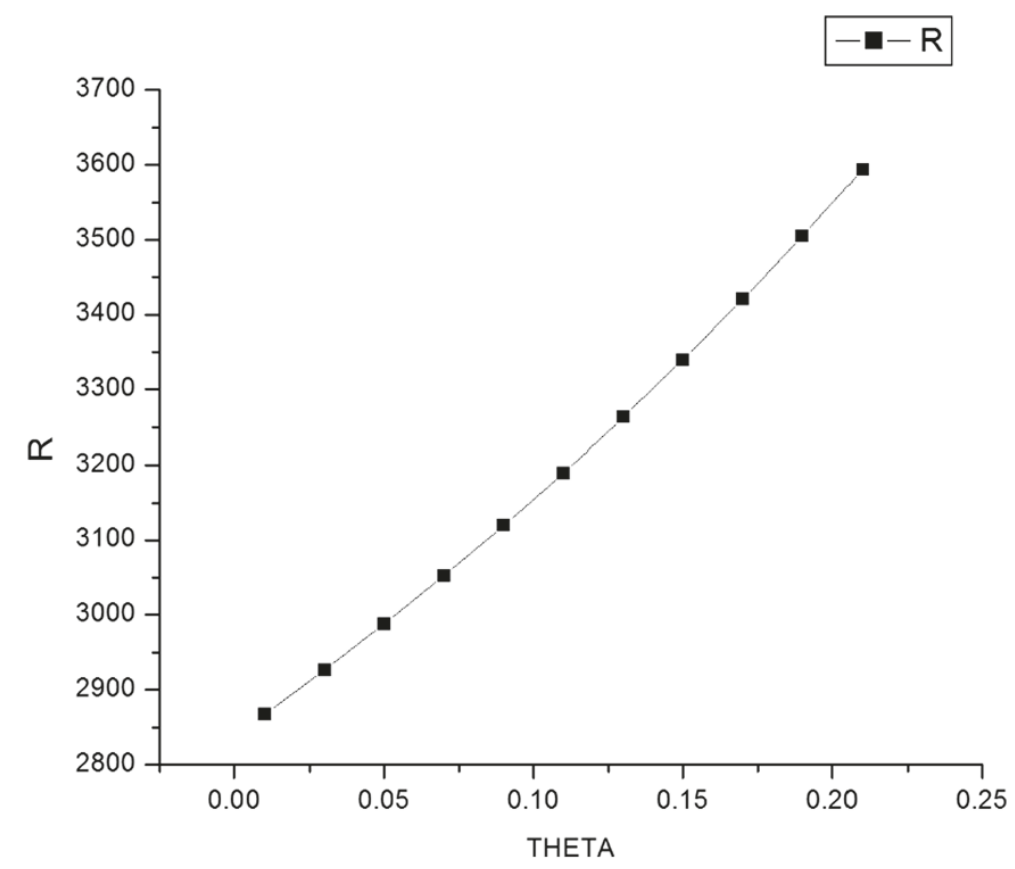

Figure 8 Graphical representation of change the optimum value of $R$ with theta.

rate $(P)$, and inventory level $(R)$ of supplier with respect to different parameters which are shown in Figures 4, 5, 6, 7, 8, 9, 10, 11, 12, and 13.

\section{Discussion}

From Tables 1 and 2, it is observed that profits under the integrated approach is greater than the Stakelberg approach and hence the former approach is better than the later

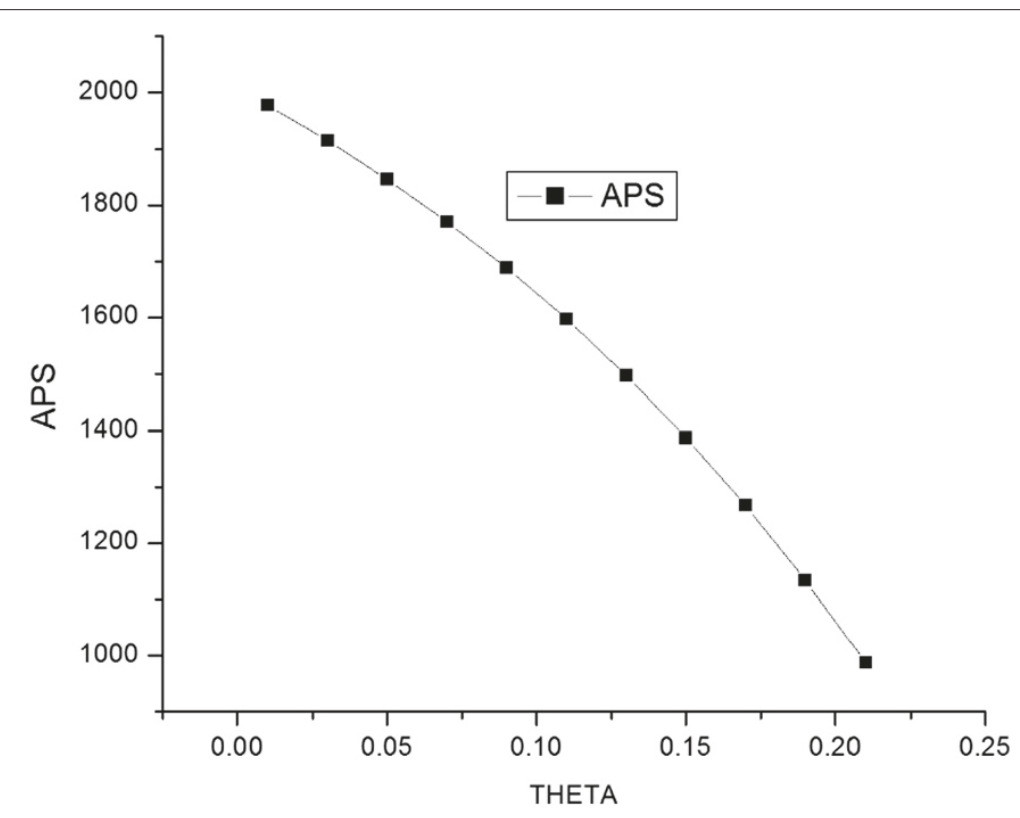

Figure 9 Graphical representation of change the optimum value of APS with theta. 


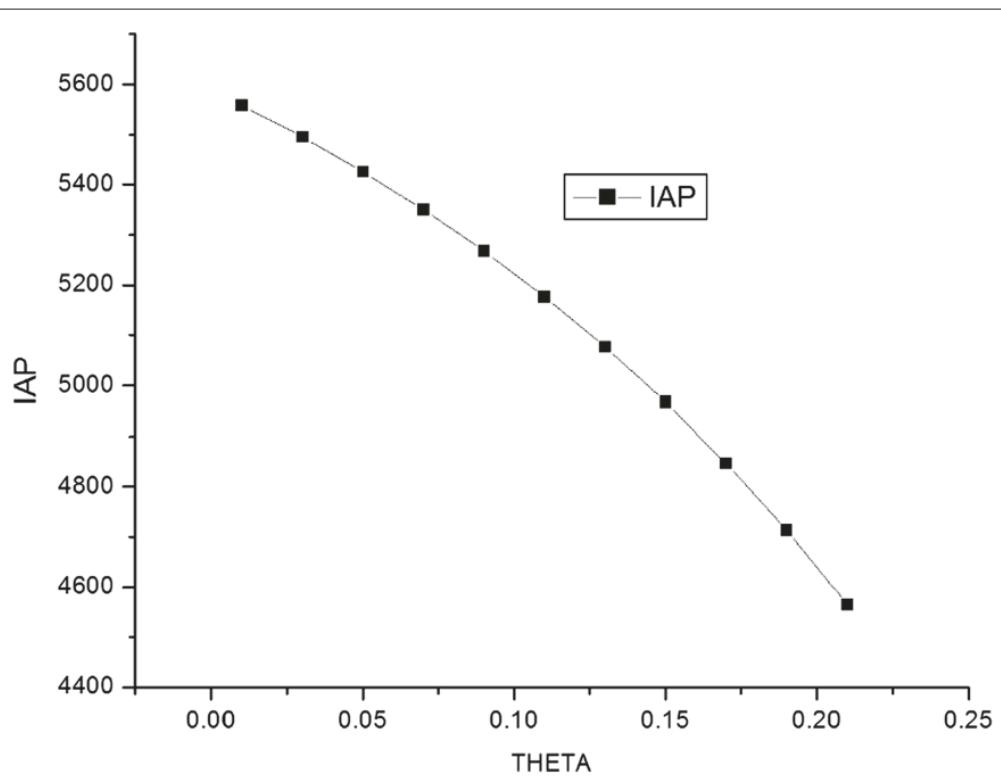

Figure 10 Graphical representation of change the optimum value of IAP with theta.

approach. The sensitivity analysis in 'Numerical examples' section shows that with increase of the reliability parameter $\alpha$, (i) the profits of the supplier and retailer are slightly increasing (Figure 6), (ii) the values of $P$ and $R$ are gradually increasing (Figures 4 and 5) but (iii) both profits of the manufacturer (APM) and the integrated profit (IAP) are gradually decreasing (Figures 6 and 7). From Figures 8, 9, 10, 11, it is also seen that the values of APS and IAP are decreasing, but the initial amount of inventory level of supplier is

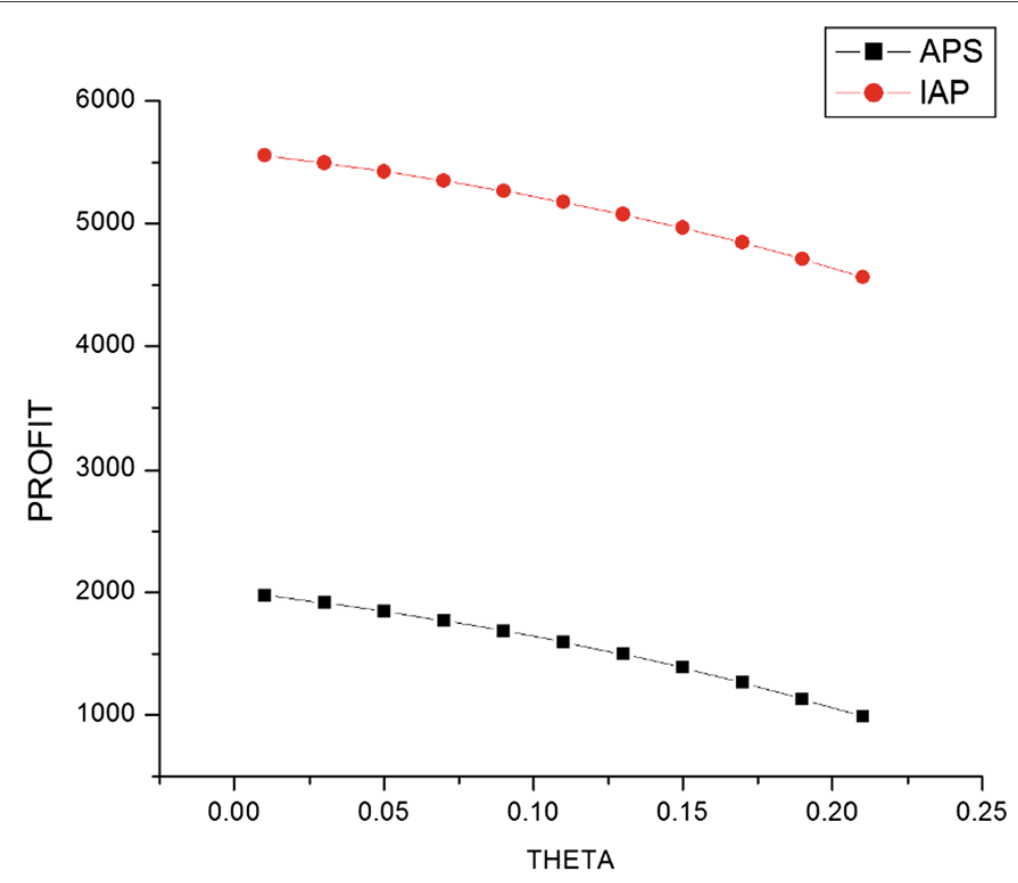

Figure 11 Graphical representation of change the optimum value of profits with theta. 


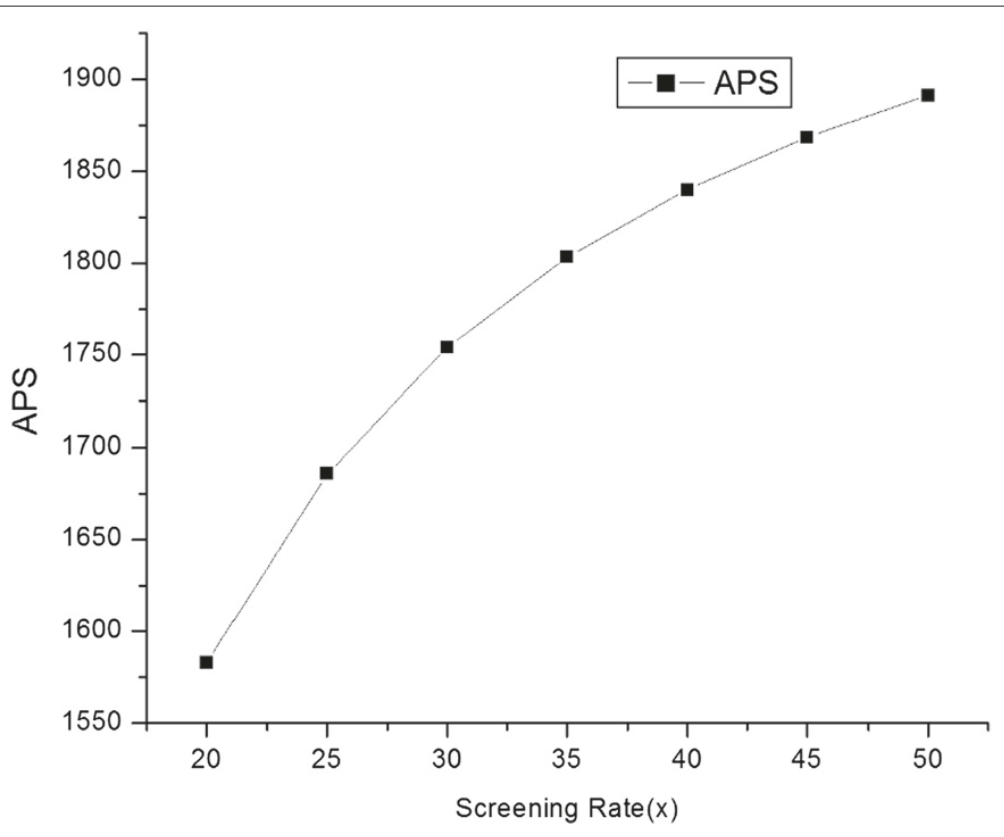

Figure 12 Graphical representation of change the optimum value of APS with $x$.

increasing with increase of $\theta$. It is also noted that the values of APS and IAP increase with the screening rate of the supplier $(x)$.

\section{Conclusions}

This paper develops a three-layer supply chain production inventory model involving supplier, manufacturer, and retailer as the members of the chain who are

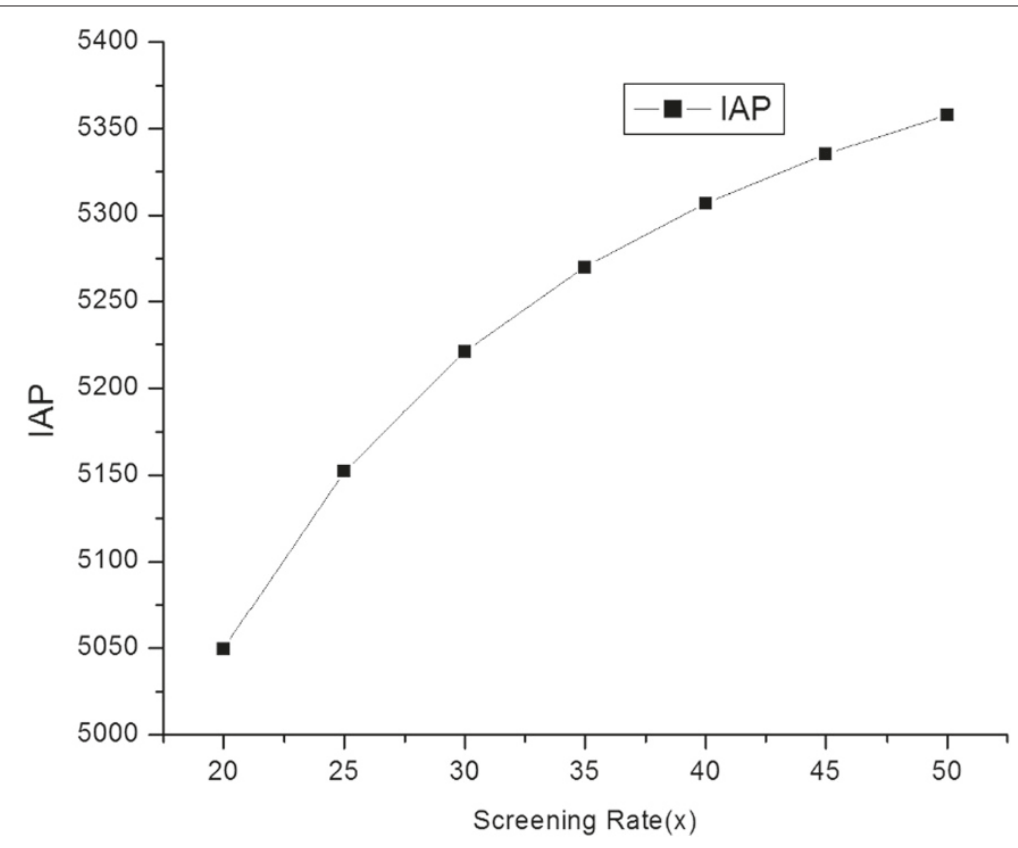

Figure 13 Graphical representation of change the optimum value of IAP with $x$. 
responsible for performing the raw materials into finished product and make them available to satisfy customers' demand in time. In comparing with the existing literature on the supply chain, the following are the main contributions in the proposed model:

Inspection cost is incurred during the production run time, and the manufacturer continuously inspects as well as separates the perfect quality items, less perfect quality items, repairable items which are transformed into perfect quality items after some rework, and reject items. Reworked cost is considered by the manufacturer to repair a certain percentage of imperfect quality items. The demand rate of the customers for perfect quality items and less perfect quality items are respectively assumed as stock dependent and selling price dependent. Here retailer have two showrooms $\mathrm{PW}_{1}$ and $\mathrm{PW}_{2}$ of finite capacities at a busy market place, and the market demands of perfect and less perfect quality items are respectively met through the showrooms $\mathrm{PW}_{1}$ and $\mathrm{PW}_{2}$. Retailer has a secondary warehouse SW of infinite capacity, away from busy market place, to store the excess amount perfect quality items from where the items are continuously transferred to the showroom. It is considered that the holding cost per unit per unit time at SW is less than the holding cost at $\mathrm{PW}_{1}$ per unit per unit time. The repairing costs of corrective and preventive maintenance should also be considered, as these costs increase the unit production cost. Inventory and production decisions are made at the supplier, manufacturer, and retailer levels. Actually in this paper, the coordination between production and inventory decisions has been established across the supply chain so that the integrated average profit of the chain is maximum.

\section{Appendix 1}

$$
\begin{aligned}
T= & t_{4}+\frac{1}{\beta_{1}} \log \left[\left(1-\frac{D_{\mathrm{r}}}{\alpha_{1}}\right)\left(1-e^{-\beta_{1} t_{3}}\right)\right] \\
= & t_{2}+\frac{S-W_{1}}{\alpha_{1}+\beta_{1} W_{1}}+\frac{1}{\beta_{1}} \log \left[\left(1-\frac{D_{\mathrm{r}}}{\alpha_{1}}\right)\left(1-e^{-\beta_{1} t_{3}}\right)\right] \\
= & \frac{P}{D_{\mathrm{r}}}\left[\frac{1}{\alpha}(1-\beta)\left(1-e^{-\alpha t_{1}}\right)+\beta t_{1}+\frac{S-W_{1}}{\alpha_{1}+\beta_{1} W_{1}}+\frac{1}{\beta_{1}} \log \left[\left(1-\frac{D_{\mathrm{r}}}{\alpha_{1}}\right)\left(1-e^{-\beta_{1} t_{3}}\right)\right]\right. \\
= & \frac{P}{D_{\mathrm{r}}} t_{1}+\frac{S-W_{1}}{\alpha_{1}+\beta_{1} W_{1}}+\frac{1}{\beta_{1}} \log \left[\left(1-\frac{D_{\mathrm{r}}}{\alpha_{1}}\right)\left(1-e^{-\beta_{1} t_{3}}\right)\right] \\
= & \frac{1-\theta}{D_{\mathrm{r}}} R+t_{0} . \\
\operatorname{APS}(R, P)= & \frac{1}{T}\left[w_{\mathrm{s}}(R-R \theta)+w_{\mathrm{s}}^{\prime} R \theta-\left(A_{\mathrm{s}}+c_{\mathrm{s}} R\right)-s_{\mathrm{c}} R-h_{\mathrm{s}}\left[\frac{(R-R \theta) t_{1}}{2}+\frac{R^{2} \theta}{x}\right]-I_{\mathrm{cs}}\left(T-t_{1}\right)\right] \\
= & \frac{1}{T}\left[-\left(A_{\mathrm{s}}+I_{\mathrm{cs}} t_{0}\right)+\left[w_{\mathrm{s}}(1-\theta)+w_{\mathrm{s}}^{\prime}-c_{\mathrm{s}}-s_{\mathrm{c}}-\frac{I_{\mathrm{cs}}(1-\theta)}{D_{\mathrm{r}}}\right] R+I_{\mathrm{cs}}(1-\theta) \frac{R}{P}\right. \\
& \left.-h_{\mathrm{s}}(1-\theta)^{2} \frac{R^{2}}{2 P}-\frac{h_{\mathrm{s}} \theta}{x} R^{2}\right] \\
= & \frac{1}{T}\left[-Z_{0 s}+Z_{1 s} R+Z_{2 s} \frac{R}{P}-Z_{3 s} \frac{R^{2}}{2 P}-Z_{4 s} R^{2}\right] .
\end{aligned}
$$

where $Z_{0 s}=\left(A_{\mathrm{s}}+I_{\mathrm{cs}} t_{0}\right) Z_{1 s}=\left[w_{\mathrm{s}}(1-\theta)+w_{\mathrm{s}}^{\prime}-c_{\mathrm{s}}-s_{\mathrm{c}}-\frac{I_{\mathrm{cs}}(1-\theta)}{D_{\mathrm{r}}}\right]$

$$
Z_{2 s}=I_{\mathrm{cs}}(1-\theta), \quad Z_{3 s}=h_{\mathrm{s}}(1-\theta)^{2}, Z_{4 s}=\frac{h_{\mathrm{s}} \theta}{x} \text {. }
$$


Manna et al. Journal of Uncertainty Analysis and Applications 2014, 2:17

Page 27 of 31

http://www.juaa-journal.com/content/2/1/17

$$
\begin{aligned}
\operatorname{APM}(R, P)= & \frac{1}{T}\left[\left(s_{\mathrm{m}} D_{\mathrm{r}} t_{2}+s_{\mathrm{m}}^{\prime} D_{\mathrm{r}}^{\prime} t_{2}^{\prime}\right)-\left\{w_{\mathrm{s}}+C(P)+I_{\mathrm{sm}}\right\} P t_{1}-r_{\mathrm{cm}}\left\{-\frac{P \beta}{\alpha}\left(1-e^{-\alpha t_{1}}\right)+P \beta t_{1}\right\}\right. \\
& -A_{\mathrm{m}}-h_{\mathrm{m}}\left\{\frac{P}{\alpha}(1-\beta) t_{1}-\frac{P}{\alpha^{2}}(1-\beta)\left(1-e^{-\alpha t_{1}}\right)+P \beta \frac{t_{1}^{2}}{2}-D_{\mathrm{r}} \frac{t_{2}^{2}}{2}\right\} \\
& -I_{\mathrm{cm}}\left(T-t_{2}\right)-h_{\mathrm{m}}^{\prime}\left\{\frac{P}{\alpha^{2}} \gamma(1-\beta)\left(1-e^{-\alpha t_{1}}\right)-\frac{\gamma}{\alpha}(1-\beta) P t_{1}\right. \\
& \left.+\left(\gamma(1-\beta) P-D_{\mathrm{r}}^{\prime} \frac{t_{1}^{2}}{2}+\frac{D_{\mathrm{r}}^{\prime}}{2}\left(t_{1}-t_{2}^{\prime}\right)^{2}\right\}\right] \\
= & \frac{1}{T}\left[\left(s_{\mathrm{m}}+\frac{I_{\mathrm{cm}}}{D_{\mathrm{r}}}\right)\left\{\frac{P}{\alpha}(1-\beta)\left(1-e^{-\alpha t_{1}}\right)+P \beta t_{1}\right\}-\left\{w_{\mathrm{s}}+C(P)+I_{\mathrm{sm}}\right\} P t_{1}-A_{\mathrm{m}}\right. \\
& +s_{\mathrm{m}}^{\prime}\left\{\frac{-P \gamma}{\alpha}(1-\beta)\left(1-e^{-\alpha t_{1}}\right)+P \gamma(1-\beta) t_{1}\right\}-r_{\mathrm{cm}}\left[-\frac{P \beta}{\alpha}\left(1-e^{-\alpha t_{1}}\right)+P \beta t_{1}\right] \\
& -I_{\mathrm{cm}} T-h_{\mathrm{m}}\left\{\frac{P}{\alpha}(1-\beta) t_{1}-\frac{P}{\alpha^{2}}(1-\beta)\left(1-e^{-\alpha t_{1}}\right)+P \beta \frac{t_{1}^{2}}{2}-D_{\mathrm{r}} \frac{t_{2}^{2}}{2}\right\} \\
- & h_{\mathrm{m}}^{\prime}\left\{\frac{P}{\alpha^{2}} \gamma(1-\beta)\left(1-e^{-\alpha t_{1}}\right)-\frac{\gamma}{\alpha}(1-\beta) P t_{1}+\left(\gamma(1-\beta) P-D_{\mathrm{r}}^{\prime}\right) \frac{t_{1}^{2}}{2}\right. \\
& \left.\left.+\frac{D_{\mathrm{r}}^{\prime}}{2}\left(t_{1}-t_{2}^{\prime}\right)^{2}\right\}\right]
\end{aligned}
$$

$$
\begin{aligned}
= & \frac{1}{T}\left[\left(s_{\mathrm{m}}+\frac{I_{\mathrm{cm}}}{D_{\mathrm{r}}}-w_{\mathrm{s}}-I_{\mathrm{sm}}-R_{1}\right) P t_{1}-I_{\mathrm{cm}} T-A_{\mathrm{m}}-G t_{1}-H P^{2} t_{1}\right. \\
& +\left[s_{\mathrm{m}}^{\prime}(1-\beta) \alpha \gamma-\left(s_{\mathrm{m}}+\frac{I_{\mathrm{cm}}}{D_{\mathrm{r}}}\right)(1-\beta) \alpha-h_{\mathrm{m}}-r_{\mathrm{cm} \alpha \beta}\right] \frac{P t_{1}^{2}}{2} \\
& \left.+\frac{h_{\mathrm{m}}}{2 D_{\mathrm{r}}} P^{2} t_{1}^{2}+h_{\mathrm{m}}^{\prime} D_{\mathrm{r}}^{\prime} t_{1}^{2}\right], \quad\left[\mathrm{using} 1-e^{-\alpha t_{1}} \approx \alpha t_{1}\right] \\
= & \frac{1}{T}\left[-I_{\mathrm{cm}} t_{0}-G(1-\theta) \frac{R}{P}+\left(s_{\mathrm{m}}+\frac{I_{\mathrm{cm}}}{D_{\mathrm{r}}}-w_{\mathrm{s}}-I_{\mathrm{sm}}-R_{1}-\frac{I_{\mathrm{cm}}}{D_{\mathrm{r}}}\right)(1-\theta) R\right. \\
& -H(1-\theta) P R^{2}+\left\{s_{\mathrm{m}}^{\prime}(1-\beta) \alpha \gamma-\left(s_{\mathrm{m}}+\frac{I_{\mathrm{cm}}}{D_{\mathrm{r}}}\right)(1-\beta) \alpha-h_{\mathrm{m}}-r_{\mathrm{cm} \alpha \beta}\right\}(1-\theta)^{2} \frac{R^{2}}{2 P} \\
& \left.+\frac{h_{\mathrm{m}}}{2 D_{\mathrm{r}}}(1-\theta)^{2} R^{2}+h_{\mathrm{m}}^{\prime} D_{\mathrm{r}}^{\prime}(1-\theta)^{2} \frac{R^{2}}{P^{2}}\right], \quad[\mathrm{using}(3)] \\
= & \frac{1}{T}\left[-Z_{0 m}-Z_{1 m} \frac{R}{P}+Z_{2 m} R+Z_{3 m} \frac{R^{2}}{P^{2}}+Z_{4 m} \frac{R^{2}}{P}+Z_{5 m} R^{2}-Z_{6 m} P R^{2}\right] .
\end{aligned}
$$

where $Z_{0 m}=I_{\mathrm{cm}} t_{0}-A_{\mathrm{m}} Z_{1 m}=G(1-\theta)$

$$
\begin{aligned}
& Z_{2 m}=\left(s_{\mathrm{m}}-w_{\mathrm{s}}-I_{\mathrm{sm}}-R_{1}\right)(1-\theta), Z_{3 m}=h_{\mathrm{m}}^{\prime} D_{\mathrm{r}}^{\prime}(1-\theta)^{2} \\
& Z_{4 m}=\left[s_{\mathrm{m}}^{\prime}(1-\beta) \alpha \gamma-\left(s_{\mathrm{m}}+\frac{I_{\mathrm{cm}}}{D_{\mathrm{r}}}\right)(1-\beta) \alpha-h_{\mathrm{m}}-r_{\mathrm{cm} \alpha \beta}\right] \frac{(1-\theta)^{2}}{2} \\
& Z_{5 m}=\frac{h_{\mathrm{m}}}{2 D_{\mathrm{r}}}(1-\theta)^{2}, \quad Z_{6 m}=H(1-\theta) .
\end{aligned}
$$




$$
\begin{aligned}
\operatorname{APR}(R, P)= & \frac{1}{T}\left[\left(s_{\mathrm{r}}-c_{\mathrm{tp}}\right)\left[\left(\alpha_{1}+\beta_{1} W_{1}\right)\left(t_{4}-t_{3}\right)+D_{\mathrm{r}} t_{3}-\frac{D_{\mathrm{r}}}{\beta_{1}}+\frac{D_{\mathrm{r}}-\alpha_{1}}{\beta_{1}} e^{-\beta_{1} t_{3}}+\frac{\alpha_{1}}{\beta_{1}} e^{-\beta_{1}\left(t_{4}-T\right)}\right]\right. \\
& +\left(s_{\mathrm{r}}^{\prime}-c_{\mathrm{tp}}^{\prime}\right) D_{\mathrm{r}}^{\prime} t_{2}^{\prime}-\frac{h_{\mathrm{rs}}}{2}\left[\left(D_{\mathrm{r}}-\left(\alpha_{1}+\beta_{1} W_{1}\right)\right)\left(t_{2}-t_{3}\right)^{2}+\left(\alpha_{1}+\beta_{1} W_{1}\right)\left(t_{2}-t_{4}\right)^{2}\right] \\
& -h_{\mathrm{r}}\left\{\frac{D_{\mathrm{r}}-\alpha_{1}}{\beta_{1}}\left(t_{3}+\frac{e^{-\beta_{1} t_{3}}}{\beta_{1}}-\frac{1}{\beta_{1}}\right)+W_{1}\left(t_{4}-t_{3}\right)-\frac{\alpha_{1}}{\beta_{1}}\left\{\left(T-t_{4}\right)+\frac{1}{\beta_{1}}-\frac{e^{-\beta_{1}\left(t_{4}-T\right)}}{\beta_{1}}\right\}\right\} \\
& -\frac{h_{\mathrm{r}}^{\prime}}{2}\left\{\left(D_{\mathrm{r}}^{\prime}-D_{\mathrm{c}}^{\prime}\right) t_{2}^{\prime 2}+D_{\mathrm{c}}^{\prime}\left(t_{2}^{\prime}-T^{\prime}\right)^{2}\right\}-A_{\mathrm{r}}-A_{\mathrm{r}}^{\prime} \\
& -s_{\mathrm{m}}\left\{\frac{P}{\alpha}(1-\beta) t_{1}-\frac{P}{\alpha^{2}}(1-\beta)\left(1-e^{-\alpha t_{1}}\right)+(P \beta) \frac{t_{1}^{2}}{2}-D_{\mathrm{r}}^{\prime} \frac{t_{2}^{2}}{2}\right\} \\
& \left.-s_{\mathrm{m}}^{\prime}\left\{\frac{P \gamma}{\alpha^{2}}(1-\beta)\left(1-e^{-\alpha t_{1}}\right)-\frac{P \gamma}{\alpha}(1-\beta) t_{1}+\frac{D_{\mathrm{r}}^{\prime}}{2}\left(t_{1}-t_{2}^{\prime}\right)^{2}+\left(P \gamma(1-\beta)-D_{\mathrm{r}}^{\prime}\right) \frac{t_{1}^{2}}{2}\right\}\right] \\
= & \frac{1}{T}\left[Z_{0 r}+Z_{1 r} R+Z_{2 r} \frac{R^{2}}{2 P}+Z_{3 r} R^{2}+Z_{4 r} \frac{R^{3}}{P}+Z_{5 r} \frac{R^{4}}{P^{2}}\right] .
\end{aligned}
$$

[using (3), (7), (15) and $\left.1-e^{-\alpha t_{1}} \approx \alpha t_{1}\right]$

where $Z_{0 r}=-h_{\mathrm{rs}}\left[\frac{\left(s-W_{1}\right)^{2}}{2\left(D_{\mathrm{r}}-\left(\alpha_{1}+\beta_{1} W_{1}\right)\right)}+\frac{\left(s-W_{1}\right)^{2}}{2\left(\alpha_{1}+\beta_{1} W_{1}\right)}\right]-A_{\mathrm{r}}-A_{\mathrm{r}}^{\prime}$

$-h_{\mathrm{r}}\left[-\frac{\alpha_{1}}{\beta_{1}^{2}} \log \left(1+\frac{\beta_{1} W_{1}}{\alpha_{1}}\right)-\frac{1}{\beta_{1}}\left(\frac{D_{\mathrm{r}}-\alpha_{1}}{\beta_{1}}-\frac{D_{\mathrm{r}} W_{1}}{\alpha_{1}+\beta_{1} W_{1}}\right) \log \left(1-\frac{W_{1} \beta_{1}}{D_{\mathrm{r}}-\alpha_{1}}\right)\right]$

$Z_{1 r}=\left(s_{\mathrm{r}}-c_{\mathrm{tp}}-\frac{h_{\mathrm{r}} W_{1}}{\alpha_{1}+\beta_{1} W_{1}}\right)(1-\theta)$

$Z_{2 r}=\left[\left(s_{\mathrm{r}}^{\prime}-c_{\mathrm{tp}}^{\prime}\right) \gamma \alpha(1-\beta)+\left(s_{\mathrm{r}}-c_{\mathrm{tp}}-\frac{h_{\mathrm{r}} W_{1}}{\alpha_{1}+\beta_{1} W_{1}}\right) \alpha(1-\beta)-\frac{W_{\mathrm{m}}}{2}\right](1-\theta)^{2}$

$Z_{3 r}=\frac{s_{\mathrm{m}}}{2 D_{\mathrm{r}}}(1-\theta)^{2}, \quad Z_{4 r}=\left[\frac{s_{\mathrm{m}}^{\prime} \gamma \alpha}{2}(1-\beta)-\frac{s_{\mathrm{m}} \gamma}{2 D_{\mathrm{r}}}(1-\beta)\right](1-\theta)^{3}$

$Z_{5 r}=\left[s_{\mathrm{m}} \frac{\gamma^{2}}{4 D_{\mathrm{r}}}(1-\beta)^{2}-s_{\mathrm{m}}^{\prime} \frac{\gamma^{2} \alpha^{2}}{8 D_{\mathrm{r}}}(1-\beta)^{2}-\frac{h_{\mathrm{r}}^{\prime}}{8 D_{\mathrm{r}}^{\prime}}\left\{\left(D_{\mathrm{r}}^{\prime}-D_{\mathrm{c}}^{\prime}\right)+\frac{\left(D_{\mathrm{r}}^{\prime}-D_{\mathrm{c}}^{\prime}\right)^{2}}{D_{\mathrm{c}}^{\prime 2}}\right\} \gamma^{2} \alpha^{2}(1-\beta)^{2}\right]$.

$\mathrm{IAP}(R, P)=[\mathrm{APS}+\mathrm{APM}+\mathrm{APR}]$

$$
\begin{aligned}
= & \frac{1}{T}\left[\left(Z_{0 r}-Z_{0 m}-Z_{0 s}\right)+\left(Z_{2 s}-Z_{1 m}\right) \frac{R}{P}+\left(Z_{1 s}+Z_{1 r}+Z_{2 m}\right) R-\left(Z_{3 s}+2 Z_{4 m}+Z_{2 r}\right) \frac{R^{2}}{2 P}\right. \\
& \left.+Z_{3 m} \frac{R^{2}}{P^{2}}-Z_{6 m} P R^{2}-\left(Z_{4 s}-Z_{3 r}-Z_{5 m}\right) R^{2}+Z_{4 r} \frac{R^{3}}{P}+Z_{5 r} \frac{R^{4}}{P^{2}}\right] \\
= & \frac{1}{T}\left[Z_{0}+Z_{1} \frac{R}{P}+Z_{2} R-Z_{3} \frac{R^{2}}{2 P}+Z_{4} \frac{R^{2}}{P^{2}}-Z_{5} P R^{2}-Z_{6} R^{2}+Z_{7} \frac{R^{3}}{P}+Z_{8} \frac{R^{4}}{P^{2}}\right] .
\end{aligned}
$$

where $Z_{0}=\left(Z_{0 r}-Z_{0 m}-Z_{0 s}\right), Z_{1}=\left(Z_{2 s}-Z_{1 m}\right), Z_{2}=\left(Z_{1 s}+Z_{1 r}+Z_{2 m}\right) Z_{3}=\left(Z_{3 s}+\right.$ $\left.2 Z_{4 m}+Z_{2 r}\right), \quad Z_{4}=Z_{3 m}, Z_{5}=Z_{6 m}, Z_{7}=Z_{4 R}, Z_{8}=Z_{5 R}, Z_{6}=\left(Z_{4 s}-Z_{3 r}-Z_{5 m}\right)$.

$$
\begin{aligned}
\frac{\partial}{\partial R}(\mathrm{APM})= & \frac{1}{T}\left[-Z_{1 m} \frac{1}{P}+Z_{2 m}+2 Z_{3 m} \frac{R}{P^{2}}+2 Z_{4 m} \frac{R}{P}+2 Z_{5 m} R-2 P R Z_{6 m}\right] \\
& -\frac{(1-\theta)}{D_{\mathrm{r}} T^{2}}\left[-Z_{0 m}-Z_{1 m} \frac{R}{P}+Z_{2 m} R+Z_{3 m} \frac{R^{2}}{P^{2}}+Z_{4 m} \frac{R^{2}}{P}+Z_{5 m} R^{2}-Z_{6 m} P R^{2}\right] \\
= & \frac{(1-\theta)}{D_{\mathrm{r}} T} Z_{0 m}+\left[\frac{(1-\theta) R}{D_{\mathrm{r}} T P}-\frac{1}{P}\right] Z_{1 m}+\left[1-\frac{(1-\theta) R}{D_{\mathrm{r}} T}\right] Z_{2 m}+\left[\frac{2 R}{P^{2}}-\frac{(1-\theta) R^{2}}{D_{\mathrm{r}} T P^{2}}\right] Z_{3 m} \\
& +\left[\frac{2 R}{P}-\frac{(1-\theta) R^{2}}{D_{\mathrm{r}} T P}\right] Z_{4 m}+\left[2 R-\frac{(1-\theta) R^{2}}{D_{\mathrm{r}} T}\right] Z_{5 m}+\left[\frac{(1-\theta) R^{2} P}{D_{\mathrm{r}} T}-2 P R\right] Z_{6 m} .
\end{aligned}
$$




$$
\begin{aligned}
& \frac{\partial}{\partial P}(\mathrm{APM})=\frac{1}{T}\left[Z_{1 m} \frac{R}{P^{2}}-2 Z_{3 m} \frac{R^{2}}{P^{3}}-Z_{4 m} \frac{R^{2}}{P^{2}}-Z_{6 m} R^{2}\right] . \\
& \frac{\partial^{2}}{\partial P^{2}}(\mathrm{APM})=2\left[-Z_{1 m} \frac{R}{P^{3}}+3 Z_{3 m} \frac{R^{2}}{P^{4}}+Z_{4 m} \frac{R^{2}}{P^{3}}\right] \\
& \frac{\partial^{2}}{\partial R \partial P}(\mathrm{APM})=\frac{1}{T}\left[Z_{1 m} \frac{1}{P^{2}}-4 Z_{3 m} \frac{R}{P^{3}}-2 Z_{4 m} \frac{R}{P^{2}}-2 Z_{6 m} R\right] \\
& \frac{\partial^{2}}{\partial R^{2}}(\mathrm{APM})=\frac{2}{T}\left[Z_{3 m} \frac{1}{P^{2}}+Z_{4 m} \frac{1}{P}+Z_{5 m}-Z_{6 m} P\right] \\
& -\frac{2(1-\theta)}{D_{\mathrm{r}} T^{2}}\left[-Z_{1 m} \frac{1}{P}+Z_{2 m}+2 Z_{3 m} \frac{R}{P^{2}}+2 Z_{4 m} \frac{R}{P}+2 Z_{5 m} R-Z_{6 m} 2 P R\right] \\
& +\frac{2(1-\theta)^{2}}{D_{r}^{2} T^{3}}\left[-Z_{0 m}-Z_{1 m} \frac{R}{P}+Z_{2 m} R+Z_{3 m} \frac{R^{2}}{P^{2}}+Z_{4 m} \frac{R^{2}}{P}+Z_{5 m} R^{2}-Z_{6 m} P R^{2}\right] \\
& =\frac{1}{T}\left[-\frac{(1-\theta)^{2}}{D_{\mathrm{r}}^{2} T^{2}} Z_{0 m}+\left\{\frac{(1-\theta)}{D_{\mathrm{r}} P T}-\frac{(1-\theta)^{2} R}{D_{\mathrm{r}}^{2} T^{2} P}\right\} Z_{1 m}+\left\{\frac{(1-\theta)^{2} R}{D_{\mathrm{r}}^{2} T^{2}}-\frac{(1-\theta)}{D_{\mathrm{r}} T}\right\} Z_{2 m}\right. \\
& +\left\{\frac{(1-\theta)^{2} R^{2}}{D_{\mathrm{r}}^{2} T^{2} P^{2}}+\frac{1}{P^{2}}-\frac{2(1-\theta) R}{D_{\mathrm{r}} T P^{2}}\right\} Z_{3 m}+\left\{\frac{(1-\theta)^{2}}{D_{\mathrm{r}}^{2} T^{2} P}+\frac{1}{P}-\frac{2(1-\theta) R}{D_{\mathrm{r}} T P}\right\} Z_{4 m} \\
& \left.+\left\{1-\frac{2(1-\theta) R}{D_{\mathrm{r}} P T}+\frac{(1-\theta)^{2} R^{2}}{D_{\mathrm{r}}^{2} T^{2}}\right\} Z_{5 m}-\left\{P-\frac{2(1-\theta) P R}{D_{\mathrm{r}} T}+\frac{(1-\theta)^{2} R^{2} P}{D_{\mathrm{r}}^{2} T^{2}}\right\} Z_{6 m}\right] . \\
& \frac{\partial}{\partial R}(\text { IAP })=\frac{1}{T}\left[Z_{1} \frac{1}{P}+Z_{2}-Z_{3} \frac{R}{P}+2 Z_{4} \frac{R}{P^{2}}-2 Z_{5} P R-2 Z_{6} R+3 Z_{7} \frac{R^{2}}{P}+4 Z_{8} \frac{R^{3}}{P^{2}}\right] \\
& -\frac{(1-\theta)}{D_{\mathrm{r}} T^{2}}\left[Z_{0}+Z_{1} \frac{R}{P}+Z_{2} R-Z_{3} \frac{R^{2}}{2 P}+Z_{4} \frac{R^{2}}{P^{2}}-Z_{5} P R^{2}-Z_{6} R^{2}+Z_{7} \frac{R^{3}}{P}+Z_{8} \frac{R^{4}}{P^{2}}\right] \\
& \frac{\partial^{2}}{\partial R^{2}}(\text { IAP })=\frac{1}{T}\left[-Z_{3} \frac{1}{P}+2 Z_{4} \frac{1}{P^{2}}-2 Z_{5} P-2 Z_{6}+6 Z_{7} \frac{R}{P}+12 Z_{8} \frac{R^{2}}{P^{2}}\right] \\
& -\frac{2(1-\theta)}{D_{\mathrm{r}} T^{2}}\left[Z_{1} \frac{1}{P}+Z_{2}-Z_{3} \frac{R}{P}+2 Z_{4} \frac{R}{P^{2}}-2 Z_{5} P R-2 Z_{6} R+3 Z_{7} \frac{R^{2}}{P}+4 Z_{8} \frac{R^{3}}{P^{2}}\right] \\
& +\frac{2(1-\theta)^{2}}{D_{\mathrm{r}}^{2} T^{3}}\left[Z_{0}+Z_{1} \frac{R}{P}+Z_{2} R-Z_{3} \frac{R^{2}}{2 P}+Z_{4} \frac{R^{2}}{P^{2}}-Z_{5} P R^{2}-Z_{6} R^{2}+Z_{7} \frac{R^{3}}{P}+Z_{8} \frac{R^{4}}{P^{2}}\right] \\
& =\frac{1}{T}\left[\frac{2(1-\theta)^{2}}{D_{\mathrm{r}}^{2} T^{2}} Z_{0}+\left\{\frac{2(1-\theta)^{2} R}{D_{\mathrm{r}}^{2} T^{2} P}-\frac{2(1-\theta)}{D_{\mathrm{r} T} P}\right\} Z_{1}+\left\{\frac{2(1-\theta)^{2} R}{D_{\mathrm{r}}^{2} T^{2}}-\frac{2(1-\theta)}{D_{\mathrm{r} T}}\right\} Z_{2}\right. \\
& -\left\{1+\frac{2(1-\theta)^{2} R^{2}}{D_{\mathrm{r}}^{2} T^{2} P}-\frac{2(1-\theta) R}{D_{\mathrm{r} T} P}\right\} Z_{3}+\left\{\frac{2}{P^{2}}+\frac{2(1-\theta)^{2} R^{2}}{D_{\mathrm{r}}^{2} T^{2} P^{2}}-\frac{4(1-\theta) R}{D_{\mathrm{r} T} P}\right\} Z_{4} \\
& -\left\{2 P+\frac{2(1-\theta)^{2} P R^{2}}{D_{\mathrm{r}}^{2} T^{2}}-\frac{4(1-\theta) P R}{D_{\mathrm{r} T}}\right\} Z_{5}-\left\{2+\frac{2(1-\theta)^{2} R^{2}}{D_{\mathrm{r}}^{2} T^{2}}-\frac{4(1-\theta) R}{D_{\mathrm{r} T}}\right\} Z_{6} \\
& \left.+\left\{\frac{6 R}{P}+\frac{2(1-\theta)^{2} R^{3}}{D_{\mathrm{r}}^{2} T^{2} P}-\frac{6(1-\theta) R^{2}}{D_{\mathrm{r} T} P}\right\} Z_{7}+\left\{\frac{12 R^{2}}{P^{2}}+\frac{2(1-\theta)^{2} R^{4}}{D_{\mathrm{r}}^{2} T^{2} P^{2}}-\frac{8(1-\theta) R^{3}}{D_{\mathrm{r} T} P^{2}}\right\} Z_{8}\right] . \\
& \frac{\partial}{\partial P}(\text { IAP })=\frac{1}{T}\left[-Z_{1} \frac{R}{P^{2}}+Z_{3} \frac{R^{2}}{2 P^{2}}-2 Z_{4} \frac{R^{2}}{P^{3}}-Z_{5} R^{2}-Z_{7} \frac{R^{3}}{P^{2}}-2 Z_{8} \frac{R^{4}}{P^{3}}\right] . \\
& \frac{\partial^{2}}{\partial P^{2}}(\mathrm{IAP})=\frac{1}{T}\left[2 Z_{1} \frac{R}{P^{3}}-Z_{3} \frac{R^{2}}{P^{3}}+6 Z_{4} \frac{R^{2}}{P^{4}}+2 Z_{7} \frac{R^{3}}{P^{3}}+6 Z_{8} \frac{R^{4}}{P^{4}}\right] . \\
& \frac{\partial^{2}}{\partial R \partial P}(\text { IAP })=\frac{1}{T}\left[-Z_{1} \frac{1}{P^{2}}+Z_{3} \frac{R}{P^{2}}-4 Z_{4} \frac{R}{P^{3}}-2 Z_{5} R-3 Z_{7} \frac{R^{2}}{P^{2}}-8 Z_{8} \frac{R^{3}}{P^{3}}\right] \\
& -\frac{(1-\theta)}{D_{\mathrm{r}} T^{2}}\left[-Z_{1} \frac{R}{P^{2}}+Z_{3} \frac{R^{2}}{2 P^{2}}-2 Z_{4} \frac{R^{2}}{P^{3}}-Z_{5} R^{2}-Z_{7} \frac{R^{3}}{P^{2}}-2 Z_{8} \frac{R^{4}}{P^{3}}\right] \\
& =\frac{1}{T}\left[\left\{\frac{(1-\theta) R}{D_{\mathrm{r}} T P^{2}}-\frac{1}{P^{2}}\right\} Z_{1}+\left\{\frac{R}{P^{2}}-\frac{(1-\theta) R^{2}}{2 D_{\mathrm{r}} T P^{2}}\right\} Z_{3}+\left\{\frac{2(1-\theta) R^{2}}{D_{\mathrm{r}} T P^{3}}-\frac{4 R}{P^{3}}\right\} Z_{4}\right. \\
& \left.+\left\{\frac{(1-\theta) R^{2}}{D_{\mathrm{r}} T}-2 R\right\} Z_{5}+\left\{\frac{(1-\theta) R^{2}}{D_{\mathrm{r}} T P^{2}}-\frac{3 R^{2}}{P^{2}}\right\} Z_{7}+\left\{\frac{2(1-\theta) R^{2}}{D_{\mathrm{r}} T P^{3}}-\frac{8 R^{3}}{P^{3}}\right\} Z_{8}\right] .
\end{aligned}
$$




\section{Appendix 2}

$$
\begin{aligned}
\frac{d}{d P}(\mathrm{APM}) & =\frac{1}{T}\left[Z_{1 m} \frac{R}{P^{2}}-2 Z_{3 m} \frac{R^{2}}{P^{3}}-Z_{4 m} \frac{R^{2}}{P^{2}}-Z_{6 m} R^{2}\right] \\
\frac{d^{2}}{d P^{2}}(\mathrm{APM}) & =\frac{1}{T}\left[-Z_{1 m} \frac{R}{P^{3}}+3 Z_{3 m} \frac{R^{2}}{P^{4}}+Z_{4 m} \frac{R^{2}}{P^{3}}\right] \\
\frac{d}{d P}(\mathrm{IAP}) & =\frac{1}{T}\left[-Z_{1} \frac{R}{P^{2}}+Z_{3} \frac{R^{2}}{2 P^{2}}-2 Z_{4} \frac{R^{2}}{P^{3}}-Z_{5} R^{2}-Z_{7} \frac{R^{3}}{P^{2}}-2 Z_{8} \frac{R^{4}}{P^{3}}\right] \\
\frac{d^{2}}{d P^{2}}(\mathrm{IAP}) & =\frac{1}{T}\left[2 Z_{1} \frac{R}{P^{3}}-Z_{3} \frac{R^{2}}{P^{3}}+6 Z_{4} \frac{R^{2}}{P^{4}}+2 Z_{7} \frac{R^{3}}{P^{3}}+6 Z_{8} \frac{R^{4}}{P^{4}}\right]
\end{aligned}
$$

\section{Acknowledgements}

The authors thanks to the reviewers and members of the editorial board for their valuable comments and constructive suggestions to improve the content of this research work.

\section{Author details}

${ }^{1}$ Department of Applied Mathematics with Oceanology and Computer Programming, Vidyasagar University, Midnapore, West Bengal 721102, India. ${ }^{2}$ Department of Mathematics, Mahishadal Raj College, Mahishadal, West Bengal, 721628, India.

Received: 27 March 2014 Accepted: 17 June 2014

Published: 23 August 2014

\section{References}

1. Weng, ZK: Channel coordination and quantity discounts. Manag. Sci. 41, 1509-1522 (1999)

2. Munson, LC, Rosenblatt, JM: Coordinating a three level supply chain with quantity discounts. IIE Trans. 33, 371-384 (2001)

3. Yang, CP, Wee, MH: An arborescent inventory model in a supply chain system. Prod. Plann. Control. 12, 728-735 (2001)

4. Khouja, M: Optimizing inventory decisions in a multistage multi customer supply chain. Trans. Res. Part E. 39, 193-208 (2003)

5. Yao, Y, Evers, PT, Dresner, ME: Supply chain integration in vendor-managed inventory. Decis. Support Syst. 43, 663-674 (2007)

6. Chaharsooghi, SK, Heydari, J, Zegordi, SH: A reinforcement learning model for supply chain ordering management: an application to the beer game. Decis. Support Syst. 45, 949-959 (2008)

7. Wang, WT, Wee, HM, Tsao, HSJ: Revisiting the note on supply chain integration in vendor-managed inventory. Decis. Support Syst. 48, 419-420 (2010)

8. Salameh, MK, Jaber, MY: Economic production quantity model for items with imperfect quality. Int. J. Prod. Econ 64, 59-64 (2000)

9. Goyal, SK, Cardenas-Barron, LE: Note on: economic production quantity model for items with imperfect quality—a practical approach. Int. J. Prod. Econ. 77, 85-87 (2002)

10. $\mathrm{Yu}, \mathrm{JCP}, \mathrm{Wee}, \mathrm{HM}, \mathrm{Chen}, \mathrm{JM}$ : Optimal ordering policy for a deteriorating item with imperfect quality and partial back ordering. J. Chin. Inst. Ind. Eng. 22, 509-520 (2005)

11. Liu, JJ, Yang, P: Optimal lot-sizing in an imperfect production system with homogeneous reworkable jobs. Eur. J. Oper. Res. 91, 517-527 (1996)

12. Panda, D, Maiti, M: Multi-item inventory models with price dependent demand under flexibility and reliability consideration and imprecise space constraint: a geometric programming approach. Math. Comput. Model. 49, 1733-1749 (2009)

13. Ma, W-N, Gong, D-C, Lin, GC: An optimal production cycle time for imperfect production process with scrap. Math. Comput. Model. 52, 724-737 (2010)

14. Sana, SS: An economic production lot size model in an imperfect production system. Eur. J. Oper. Res. 201, 158-170 (2010)

15. Sana, SS: A production-inventory model of imperfect quality products in a three-layer supply chain. Decis. Support Syst. 50, 539-547 (2011)

16. Hartely, RV: Operations Research - a Managerial Emphasis, Good Year Publishing Company, California, pp. 315-317 (1976)

17. Sarma, KVS: A deterministic inventory model with two levels of storage and an optimum release rule. Opsearch 20, 175-180 (1983)

18. Dave, U: On the EOQ models with two levels of storage. Opsearch. 25, 190-196 (1988)

19. Goswami, A, Chaudhuri, KS: An economic order quantity model for items with two level of storage for a linear trend in demand. J. Oper. Res. Soc. 43, 157-167 (1992)

20. Pakkala, TPM, Achary, KK: Discrete time inventory model for deteriorating items with two warehouses. Opsearch 29, 90-103 (1992)

21. Bhunia, AK, Maiti, M: A two warehouses inventory model for deteriorating items with a linear trend in demand and shortages. Journal of Operational Research society. 49, 287-292 (2007) 
22. Benkherouf, L: A deterministic order level inventory model for deteriorating items with two storage facilities. Int. J. Prod. Econ. 48, 167-175 (1997)

23. Zhou, YW: An optimal EOQ model for deteriorating items with two warehouses and time varying demand. Mathematica Applicata. 10, 19-23 (1998)

24. Kar, SK, Bhunia, AK, Maiti, M: Deterministic inventory model with levels of storage, a linear trend in demand and a fixed time horizon. Comp. Oper. Res. 28, 1315-1331 (2001)

25. Chung, K, Huang, T: The optimal retailer's ordering policies for deteriorating items with limited storage capacity under trade credit financing. Int. J. Prod. Econ. 106, 127-146 (2007)

26. Dey, JK, Mondal, SK, Maiti, M: Two storage inventory problem with dynamic demand and interval valued lead-time over finite time horizon under inflation and time-value of money. Eur. J. Oper. Res. 185, 170-194 (2008)

27. Liang, Y, Zhou, F: A two-warehouse inventory model for deteriorating items under conditionally permissible delay in payment. Appl. Math. Model. 35, 2221-2231 (2011)

28. Hariga, M: Inventory models for multi-warehouse systems under fixed and flexible space leasing contracts. Comput. Ind. Eng. 61, 744-751 (2011)

29. Dubois, D, Prade, H: Rough fuzzy sets and fuzzy rough sets. Int. J. Gen. Syst. 17, 91-208 (1990)

30. Morsi, NN, Yakout, MM: Axiomatics for fuzzy rough sets. Fuzzy Set. Syst. 100, 327-342 (1998)

31. Radzikowska, AM, Kerre, EE: A comparative study of rough sets. Fuzzy Set. Syst. 126, 137-155 (2002)

32. Xu, J, Zhou, X: Fuzzy-Like Multiple Objective Decision Making. Springer, Berlin (2009)

33. Liu, G, Sai, Y: Invertible approximation operators of generalized rough sets and fuzzy rough sets. Inf. Sci. 180, 2221-2229 (2010)

34. Chen, D, Kwong, S, He, Q, Wang, H: Geometrical interpretation and applications of membership functions with fuzzy rough set. Fuzzy Set and Syst. 193, 122-135 (2012)

doi:10.1186/s40467-014-0017-1

Cite this article as: Manna et al:: Three-layer supply chain in an imperfect production inventory model with two

storage facilities under fuzzy rough environment. Journal of Uncertainty Analysis and Applications 2014 2:17.

\section{Submit your manuscript to a SpringerOpen ${ }^{\circ}$ journal and benefit from:}

- Convenient online submission

- Rigorous peer review

- Immediate publication on acceptance

- Open access: articles freely available online

- High visibility within the field

Retaining the copyright to your article

Submit your next manuscript at $\gg$ springeropen.com 